Article

\title{
Synthesis of Some Novel Heterocyclic and Schiff Base Derivatives as Antimicrobial Agents
}

\author{
Mohamed E. Azab ${ }^{1, *}$, Sameh A. Rizk ${ }^{1}$ and Abd El-Galil E. Amr ${ }^{2,3}$ \\ 1 Department of Chemistry, Faculty of Science, University of Ain Shams, Cairo 11566, Egypt; \\ E-Mail: samehrizk2006@gmail.com \\ 2 Pharmaceutical Chemistry Department, Drug Exploration \& Development Chair (DEDC), \\ College of Pharmacy, King Saud University, Riyadh 11451, Saudi Arabia; \\ E-Mail: aeamr1963@yahoo.com oraamr@ksu.edu.sa \\ 3 Applied Organic Chemistry Department, National Research Center, Dokki, Cairo 12622, Egypt \\ * Author to whom correspondence should be addressed; E-Mail: meazabali2015@yahoo.com \\ or meazab@sci.asu.edu.eg; Tel.: +20-112-144-1248; Fax: +20-226-028-680.
}

Academic Editor: Wim Dehaen

Received: 5 September 2015 / Accepted: 23 September 2015 / Published: 7 October 2015

\begin{abstract}
Treatment of 2,3-diaryloxirane-2,3-dicarbonitriles 1a-c with different nitrogen nucleophiles, e.g., hydrazine, methyl hydrazine, phenyl hydrazine, hydroxylamine, thiosemicarbazide, and/or 2-amino-5-phenyl-1,3,4-thiadiazole, afforded pyrazole, isoxazole, pyrrolotriazine, imidazolothiadiazole derivatives $\mathbf{2}-\mathbf{5}$, respectively. Reacting pyrazoles $\mathbf{2 a - c}$ with aromatic aldehydes and/or methyl glycinate produced Schiff's bases $\mathbf{7 a - d}$ and pyrazolo[3,4-b]-pyrazinone derivative $\mathbf{8}$, respectively. Treating $\mathbf{7}$ with ammonium acetate and/or hydrazine hydrate, furnished the imidazolopyrazole and pyrazolotriazine derivatives $\mathbf{9}$ and 10, respectively. Reaction of $\mathbf{8}$ with chloroacetic acid and/or diethyl malonate gave tricyclic compound $\mathbf{1 1}$ and triketone 12, respectively. On the other hand, compound $\mathbf{1}$ was reacted with active methylene precursors, e.g., acetylacetone and/or cyclopentanone producing adducts 14a,b which upon fusion with ammonium acetate furnished the 3-pyridone derivatives $\mathbf{1 5 a}, \mathbf{b}$, respectively. Some of newly synthesized compounds were screened for activity against bacterial and fungal strains and most of the newly synthesized compounds showed high antimicrobial activities. The structures of the new compounds were elucidated using IR, ${ }^{1} \mathrm{H}-\mathrm{NMR},{ }^{13} \mathrm{C}-\mathrm{NMR}$ and mass spectroscopy.
\end{abstract}


Keywords: oxirane; pyrazole; isoxazole; pyrazolopyrazine; Schiff bases; pyridones; antimicrobial activities

\section{Introduction}

2,3-Diaryloxirane-2,3-dicarbonitriles are well-known as important synthetic intermediates [1,2]. The reaction of such compounds with nitrogen and carbon nucleophiles produces many biologically active heteocyclic compounds. For example, substituted pyrazoles are reported to be an important class of compounds in the agricultural and medicinal chemistry fields because of their broad spectrum biological activities [3-6] and they also have anti-cancer effects [7]. Imidazole derivatives act as potent and selective neuropeptide Y Y5 receptor antagonists, having antifungal and antibacterial activity and are used as potential tuberculostatic agents [8-10]. On the other hand, isooxazole derivatives have antifungal activity against Candida albicans, immunological and immunotropic activities [11-13]. Schiff bases have remarkable complex-forming properties and serve as excellent chelating ligands and have been used as analytical reagents for the spectrophotometric determination of metal ions $[14,15]$. For the abovementioned properties, and in continuation of our program in synthesis of biologically active heterocyclic compounds [16-26], we decided to use 2,3-diaryloxirane-2,3-dicarbonitrile derivatives 1a-c as a key starting material for the purpose of preparing some novel heterocyclic compounds by reaction with different nitrogen and carbon nucleophiles whereby we synthesized pyrazoles, oxazoles, fused pyrazoles and pyridines, and then study their antimicrobial activity. Most of the newly synthesized compounds were screened in vitro for their antimicrobial activities against different strains of bacteria and fungi. Some of the compounds such as compounds $\mathbf{7 a}$ and $\mathbf{7 b}$ showed high antibacterial activity similar to or higher than that of the reference compounds, suggesting that they may find use as antibacterial agents.

\section{Results and Discussion}

\subsection{Chemistry}

The 2,3-diaryloxirane-2,3-dicarbonitrile derivatives 1a-c were allowed to react with different nitrogen binucleophiles. Thus, compounds 1a-c were treated with hydrazine derivatives (hydrazine hydrate, methyl hydrazine and/or phenyl hydrazine), hydroxylamine hydrochloride, thiosemicarbazide, and/or 2-amino-5-aryl-1,3,4-thiadiazole to afford 3-amino-1-substituted-5,5-diaryl-1H-pyrazol-4(5H)ones 2a-e, 3-amino-5,5-diarylisoxazol-4(5H)-ones 3a-c, pyrrolo[2,3-e][1,2,4]triazine-3(2H)-thione (4) and imidazo[2,1-b][1,3,4]thiadiazol-5(6H)-one (5), respectively (Scheme 1). 


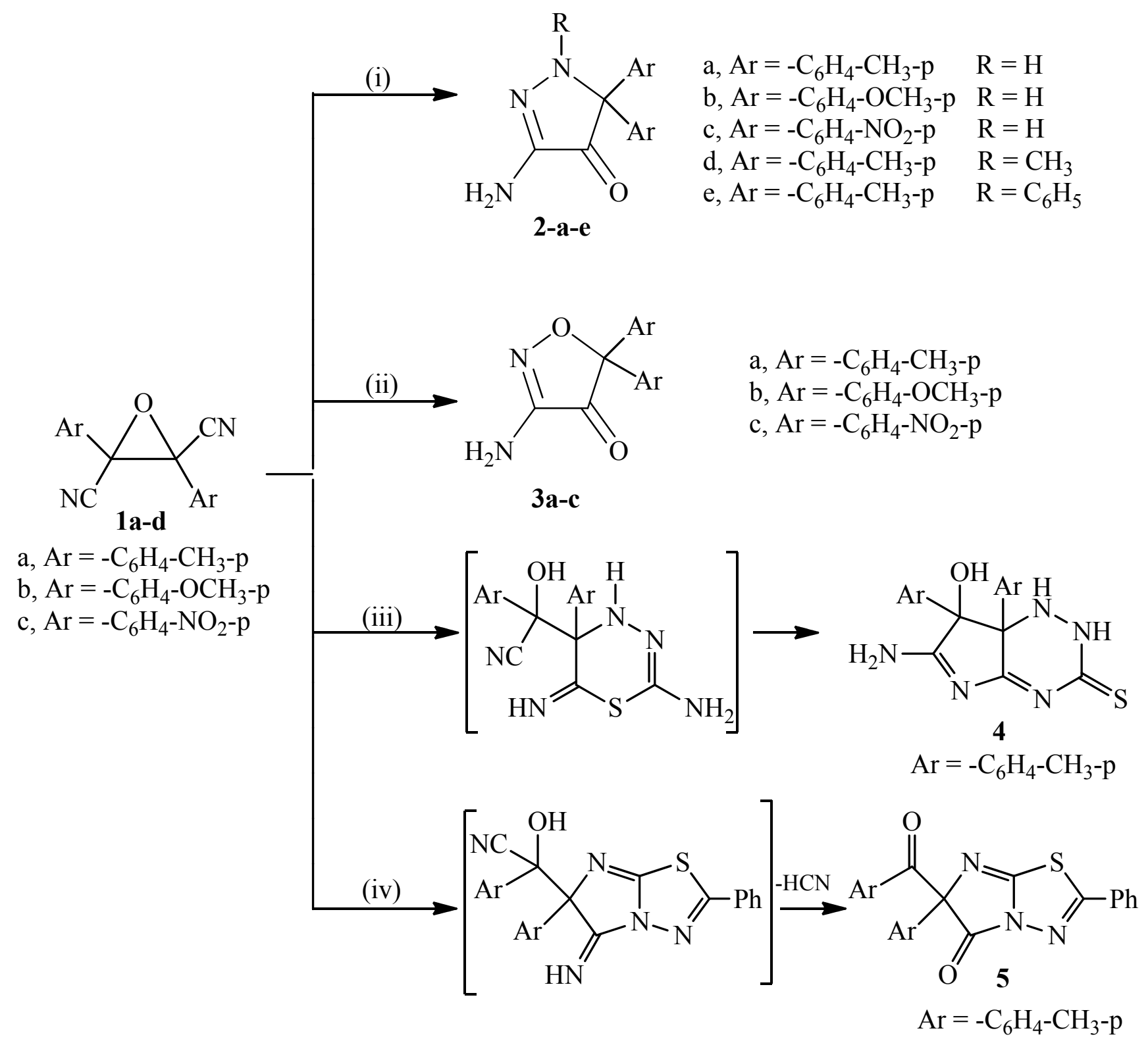

Reagents and Conditions: (i) $\mathrm{N}_{2} \mathrm{H}_{4} /$ ethanol/reflux 6 h, 60\%-76\%; (ii) $\mathrm{NH}_{2} \mathrm{OH} /$ pyridine/reflux 5 h, 68\%-77\%;

(iii) $\mathrm{NH}_{2} \mathrm{NHCSNH}_{2} /$ ethanol/reflux, 77\%; (iv) 2-amino-5-phenyl-1,3,4-thiadiazole/ethanol/ reflux 4 h, 65\%.

Scheme 1. Synthetic routes for compounds 2-5.

The speculated mechanism for the formation of compound $\mathbf{2}$ is shown in Scheme 2.

On the other hand, when compound 1 was reacted with hydrazine hydrate in boiling $n$-butanol, two products were isolated, one of them was the pyrazolone $\mathbf{2}$ and the other was identified to be the imidazolopyrazolone 6 (Scheme 3). The structure of 6 was elucidated from its ${ }^{1} \mathrm{H}-\mathrm{NMR}$ and ${ }^{13} \mathrm{C}-\mathrm{NMR}$ spectra, which indicate the presence of four aryl groups and two carbonyl groups. The postulated mechanism for the formation of compound 6 is shown in Scheme 3. 


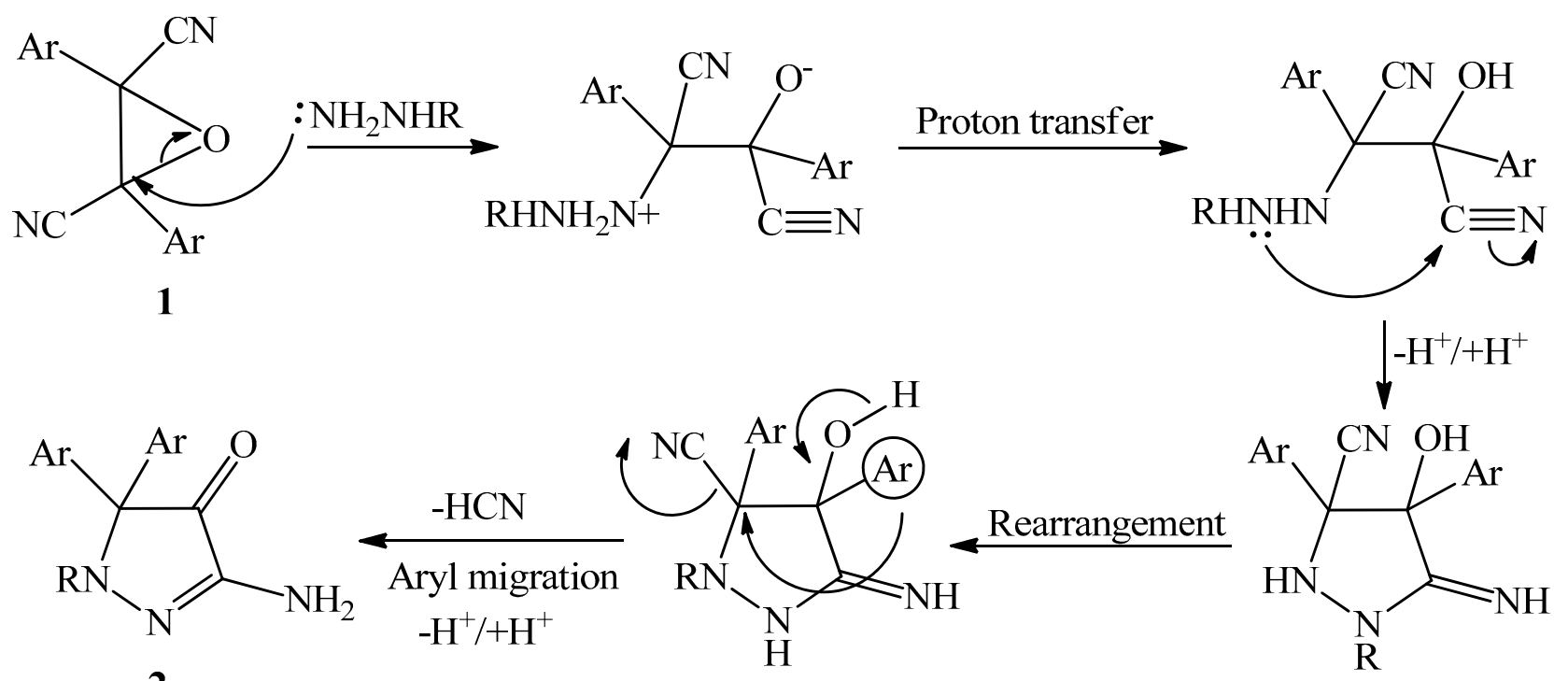

Scheme 2. Mechanism for the formation of compound 2.<smiles>N#CC1(Br)OC1(Br)Br</smiles>

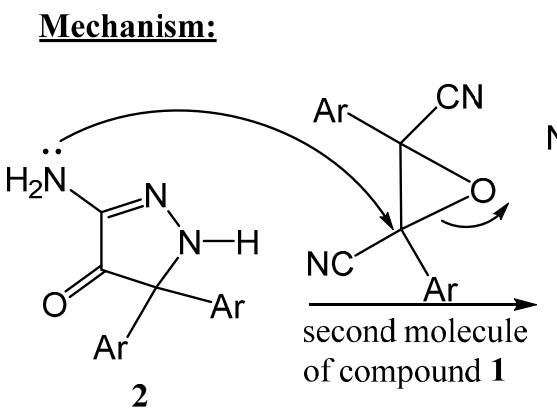

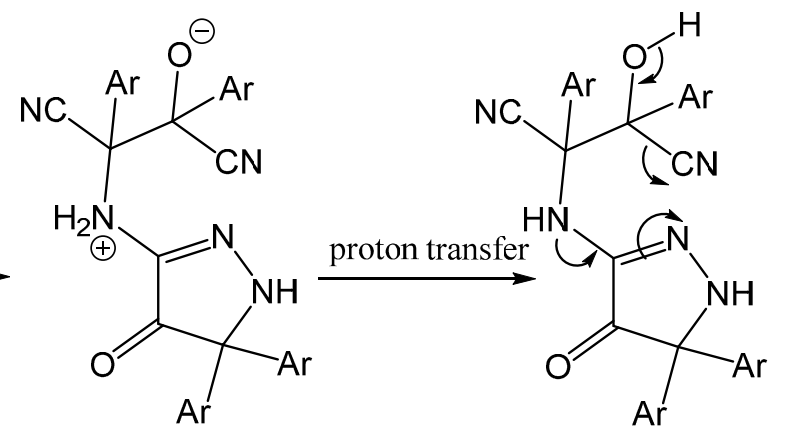

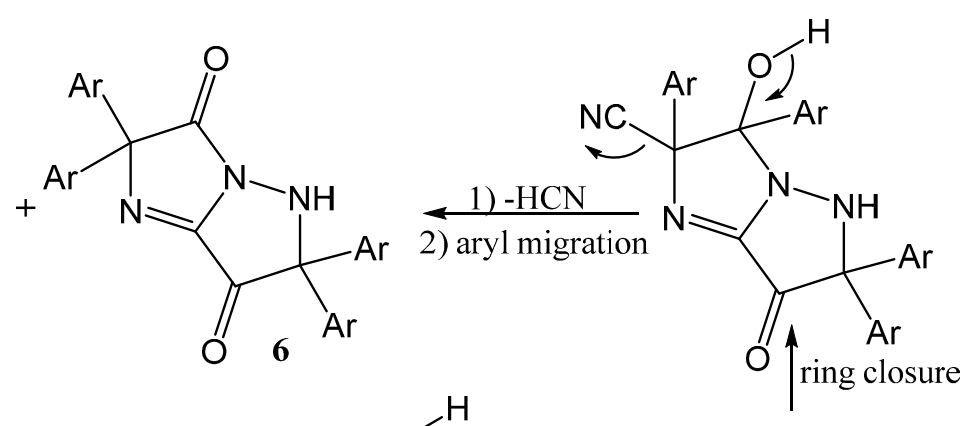

Scheme 3. Synthetic route for compound 6.

The structures of pyrazolone derivatives $\mathbf{2 a - c}$ were supported chemically by their reaction with aromatic aldehydes (namely: benzaldehyde, and p-chlorobenzaldehyde) and/or methyl glycinate, in boiling ethanol, producing the Schiff's bases $7 \mathbf{a}-\mathbf{d}$ and the pyrazolo[3,4-b]-pyrazinone derivative 8, respectively (Scheme 4). 


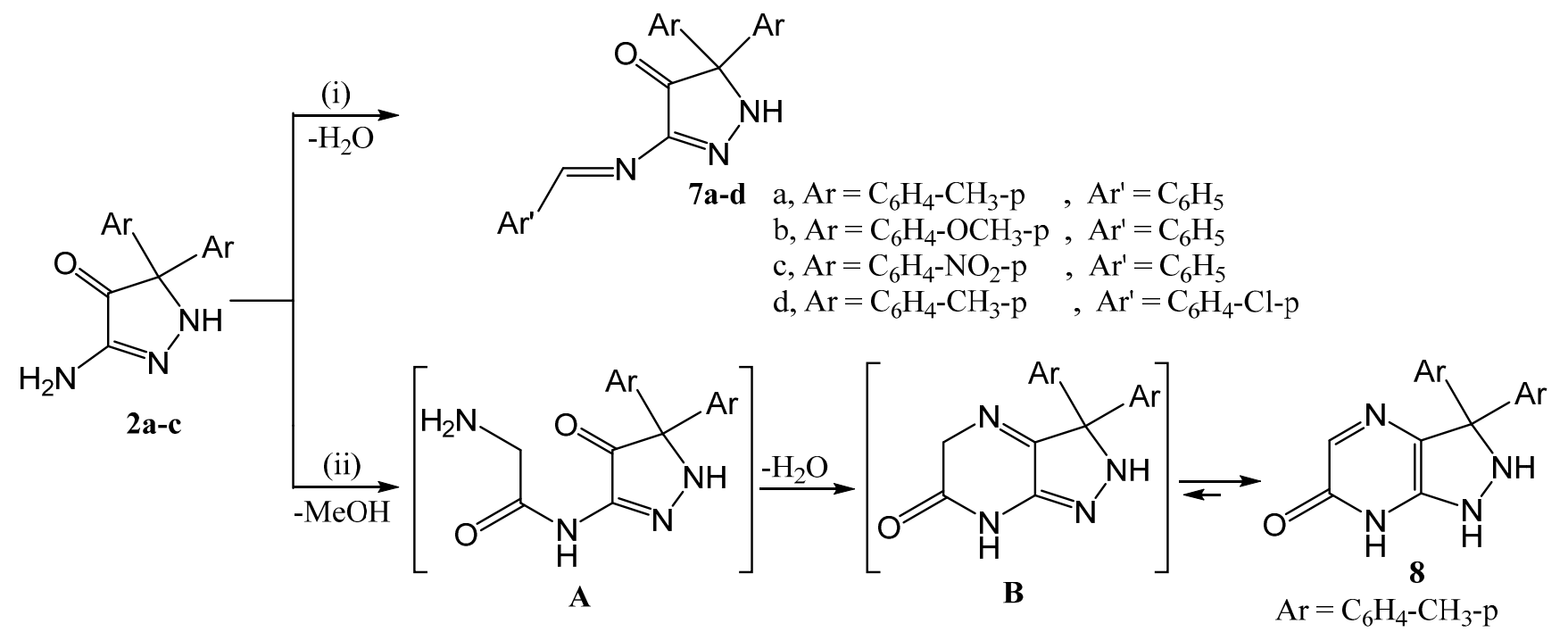

Reaction Conditions: (i) ArCHO/ethanol/reflux 4 h, 65\%-75\%; (ii) Methyl glycinate/ethanol/reflux 4 h, $70 \%$.

Scheme 4. Synthetic routes for compounds 7 and 8 .

The formation of compounds $\mathbf{7 a - c}$ takes place through condensation reactions between the carbonyl group of the aldehyde and the amino group of compound $\mathbf{2}$ (accompanied by loss of a water molecule). The reaction of $\mathbf{2}$ with methyl glycinate takes place by the attack of the pyrazole amino group on the ester group followed by the removal of a methanol molecule forming the intermediate $\mathrm{A}$, then elimination of a water molecule to form intermediate B which rearranges to the more stable form $\mathbf{8}$.

Furthermore, the Schiff's bases 7a-c were subjected to reaction with ammonium acetate (fusion at $90{ }^{\circ} \mathrm{C}$ ) and/or hydrazine hydrate (in boiling ethanol), afforded the imidazolopyrazoles 9a-c and the pyrazolo[3,4-e]1,2,4-triazine derivatives 10a-c, respectively (Scheme 5).

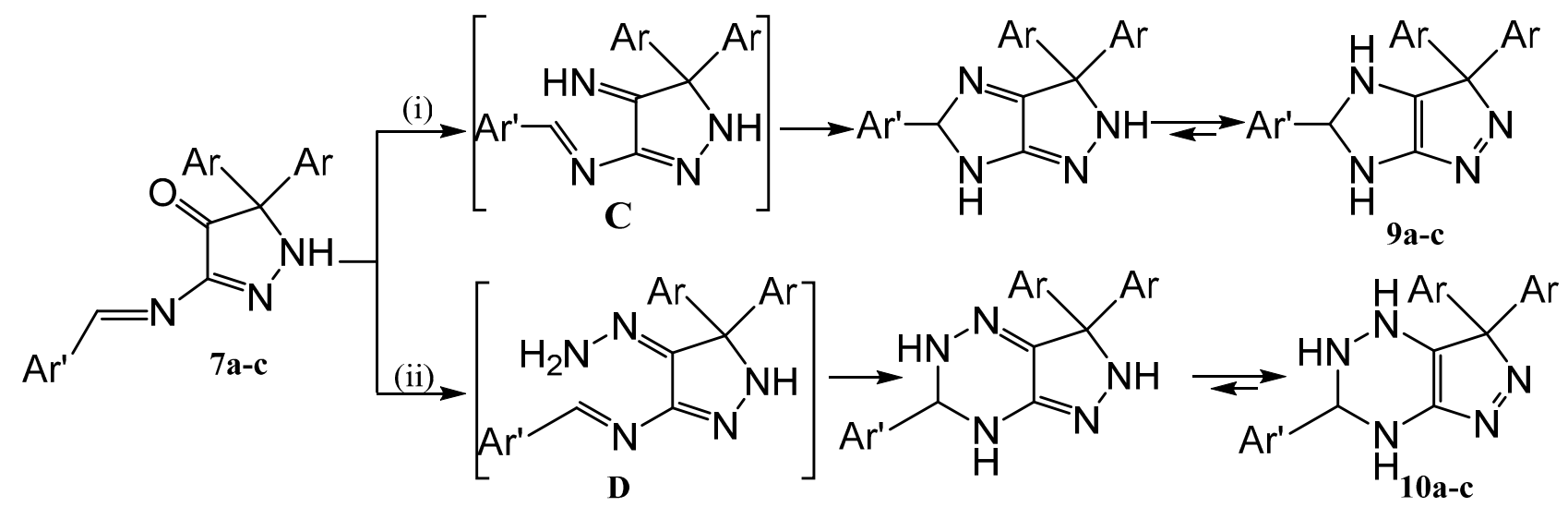

a, $\mathrm{Ar}=\mathrm{C}_{6} \mathrm{H}_{4}-\mathrm{CH}_{3}-\mathrm{p} \quad, \mathrm{Ar}^{\prime}=\mathrm{C}_{6} \mathrm{H}_{5}$

b, $\mathrm{Ar}=\mathrm{C}_{6} \mathrm{H}_{4}-\mathrm{OCH}_{3}-\mathrm{p}, \mathrm{Ar}^{\prime}=\mathrm{C}_{6} \mathrm{H}_{5}$

$\mathrm{d}, \mathrm{Ar}=\mathrm{C}_{6} \mathrm{H}_{4}-\mathrm{NO}_{2}-\mathrm{p} \quad, \mathrm{Ar}^{\prime}=\mathrm{C}_{6} \mathrm{H}_{5}$

Reaction Conditions: (i) Ammonium acetate/fusion at $90{ }^{\circ} \mathrm{C} / 3 \mathrm{~h}, 66 \%-74 \%$; (ii) $\mathrm{N}_{2} \mathrm{H}_{4} \cdot \mathrm{H}_{2} \mathrm{O} /$ ethanol/reflux $6 \mathrm{~h}, 68 \%-72 \%$.

Scheme 5. Synthetic routes for compounds 9 and $\mathbf{1 0 .}$ 
Compounds 9a-c were formed by the formation of the imine $\mathrm{C}$ (by condensation of ammonia and the carbonyl group) followed by ring closure via the attack of the imino group on the $\mathrm{C}=\mathrm{N}$ moiety of the Schiff's base. Similarly, formation of compounds $\mathbf{1 0 a}-\mathbf{c}$ occurs by the formation of the hydrazone D followed by ring closure via the attack of the amino group on the Schiff's base $C=N$.

Treatment of the pyrazolopyrazinone derivative 8 with chloroacetic acid in the presence of phosphorus oxychloride furnished the tricyclic compound 11, while reaction of diethyl malonate with $\mathbf{8}$ in boiling ethanol produced the triketone 12, which upon refluxing with benzaldehyde in ethanol, afforded the chalcone $\mathbf{1 3}$ (Scheme 6).

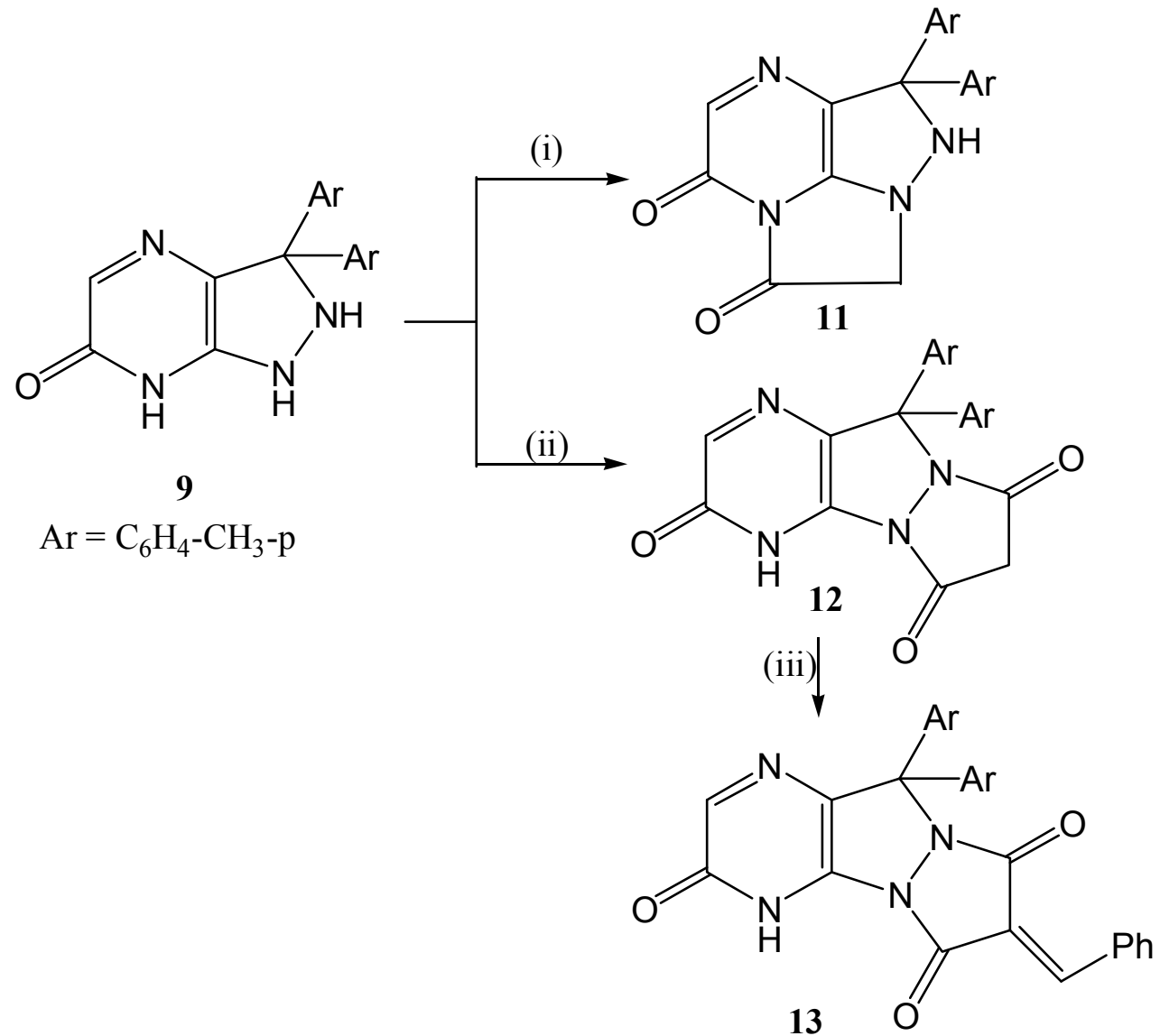

Reaction Conditions: (i) $\mathrm{ClCH}_{2} \mathrm{CO}_{2} \mathrm{H} / \mathrm{POCl}_{3} /$ reflux 2 h, 55\%; (ii) $\mathrm{CH}_{2}\left(\mathrm{CO}_{2} \mathrm{C}_{2} \mathrm{H}_{5}\right) / 2$ ethanol/reflux 4 h, 57\%; (iii) $\mathrm{PhCHO} /$ ethanol/reflux 6 h, 24\%.

Scheme 6. Synthetic routes for compounds 11-13.

The present investigation was extended to explore the reactivity of the oxirane derivative 1a towards some carbon nucleophiles. Thus, when compound 1a was refluxed in ethanol with active methylene compounds (namely: acetylacetone, and/or cyclohexanone) in the presence of $50 \%$ aqueous $\mathrm{NaOH}$ (as a catalyst), the adducts $\mathbf{1 4 a}, \mathbf{b}$ were obtained. Cyclization of $\mathbf{1 4 a}, \mathbf{b}$, by fusion with ammonium acetate furnished the 3-pyridone derivatives 15a,b (Scheme 7). 
<smiles>N#CC1([Al])OC1([Al])C#N</smiles><smiles>[C]1CCCC1</smiles><smiles>[Y]C(=O)C([X])C(C)([AlH2])C(O)([AlH2])[AlH2]</smiles><smiles>[Y]C1=C([X])C(Br)(Br)C(=O)C(N)=N1</smiles>

14a,b $\mathrm{Ar}=\mathrm{C}_{6} \mathrm{H}_{4}-\mathrm{CH}_{3}-\mathrm{p} \quad$ Active methylene a<smiles>CC(=O)CC(C)=O</smiles>
X Y $\mathrm{COCH}_{3} \quad \mathrm{CH}_{3}$ 15a,b<smiles>O=C1CCCC1</smiles><smiles>CCCC</smiles>

- $\mathrm{CH}_{2} \mathrm{CH}_{2} \mathrm{CH}_{2}$ -

Reaction Conditions: (i) Active methylene/ $\mathrm{NaOH} /$ ethanol/reflux $3 \mathrm{~h}, 67 \%-70 \%$; (ii) Ammonium acetate/fusion at $150{ }^{\circ} \mathrm{C} / 2 \mathrm{~h}, 65 \%-74 \%$.

Scheme 7. Synthetic routes for compounds 14 and 15.

A plausible mechanism for the formation of compounds $\mathbf{1 5 a}, \mathbf{b}$ is illustrated in Scheme 8 .<smiles>[Y]C(=O)C([X])(C#N)C(C)([Al])C(N)=O</smiles>

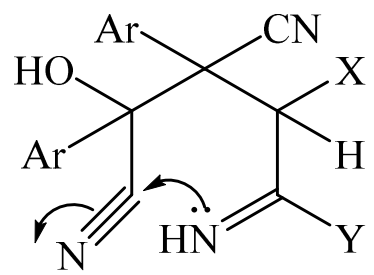

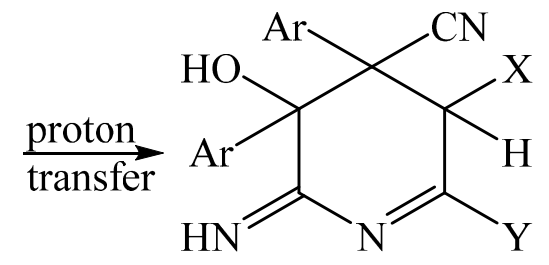

14<smiles>[R]C1=C([R])C([18F])([Al])C(=O)C(N)=N1</smiles>

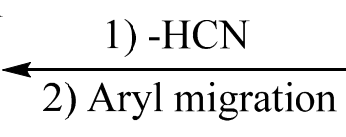<smiles>[Y]C1=C([X])C([3H])(C#N)C([3H])(O[TlH])C(N)=N1</smiles>
15

Scheme 8. Mechanism for the formation of compounds $15 \mathbf{a}, \mathbf{b}$.

\subsection{Antimicrobial Activity}

Most of the newly synthesized compounds were screened in vitro for their antimicrobial activities against different strains of bacteria and fungi. The microorganisms used were Staphylococcus aureus (Gram positive), Escherichia coli, Pseudomonas aeruginosa (Gram negative) and Candida albicans (yeast) by using the agar diffusion method [27] to select the most potent compounds.

One mg of each compound was dissolved in dimethyl sulfoxide (DMSO, $1 \mathrm{~mL}$ ) then made up to $10 \mathrm{~mL}$ with sterile water to give a concentration of $100 \mu \mathrm{g} / \mathrm{mL}$. The bacteria were maintained on nutrient agar media. The efficiency of the tested compounds was compared to that of ampicillin, streptomycin and nystatin. 
The agar media was incubated with the different tested microorganisms. After 24-48 h of incubation at $37{ }^{\circ} \mathrm{C}$, DMSO showed no inhibition zones. The diameters of the inhibition zones of the tested compounds were measured. The results are presented in Table 1.

Table 1. Antimicrobial activity of compounds $\mathbf{2}-\mathbf{1 5}$.

\begin{tabular}{ccccc}
\hline $\begin{array}{c}\text { Synthesized } \\
\text { Compounds }\end{array}$ & $\begin{array}{c}\text { Staphylococcus } \\
\text { Aureus }\end{array}$ & Escherichia Coli & $\begin{array}{c}\text { Pseudomonas } \\
\text { Aeruginosa }\end{array}$ & Candida Albicans \\
\hline $2 \mathrm{a}$ & 19 & 20 & 21 & 15 \\
$2 \mathrm{~b}$ & 19 & 16 & 21 & 15 \\
$2 \mathrm{c}$ & 17 & 18 & 16 & 11 \\
4 & 12 & 12 & 18 & 12 \\
5 & 12 & 16 & 18 & 10 \\
$6 \mathrm{a}$ & 12 & 11 & 15 & 0.0 \\
$7 \mathrm{a}$ & 18 & 23 & 22 & 15 \\
$7 \mathrm{~b}$ & 17 & 17 & 21 & 13 \\
$9 \mathrm{a}$ & 20 & 14 & 20 & 21 \\
$9 \mathrm{~b}$ & 11 & 13 & 10 & 16 \\
$10 \mathrm{a}$ & 13 & 13 & 0.0 & 11 \\
12 & 14 & 16 & 0.0 & 13 \\
13 & 16 & 17 & 19 & 12 \\
14 & 15 & 12 & 21 & 11 \\
15 & 13 & 22 & 0.0 & 0.0 \\
Ampicillin & 0.0 & 21 & 0.0 & 0.0 \\
Streptomycin & 20 & 0.0 & 0.0 & 22 \\
Nystatin & 0.0 & 0.0 & \\
\hline
\end{tabular}

Zone of inhibition measured in mm: no activity $(0.0)$, very weak activity $(<7 \mathrm{~mm})$, weak activity $(7-10)$, moderate activity $(11-15 \mathrm{~mm})$, strong activity $(>15 \mathrm{~mm})$.

The antimicrobial activity results revealed that most of the tested compounds have moderate to strong activity. The most potent compounds are $7 \mathbf{a}, 7 \mathbf{b}$ and $9 \mathbf{a}$. When these compounds were compared with the reference compounds (ampicillin, streptomycin and nystatin) we found that they have an antimicrobial activity higher or almost equal to them. On the other hand, in comparing the obtained values of antimicrobial activity for our newly synthesized Schiff bases $\mathbf{7 a}$, 7b with similar compounds which were previously reported [28,29], we found that our compounds display higher antimicrobial activity values.

\section{Experimental Section}

\subsection{General Information}

All melting points are uncorrected and were determined on a Stuart electric melting point apparatus. The microanalysis were within $\pm 0.4 \%$ of theoretical values and were carried out at the Microanalytical Centre, National Research Centre, Cairo, Egypt. IR spectra (KBr) were recorded on a FT-IR 400D infrared spectrometer (New York, USA) using the OMNIC program and are reported as frequency of absorption in $\mathrm{cm}^{-1}$. ${ }^{1} \mathrm{H}-\mathrm{NMR}$ spectra were recorded on a Bruker spectrophotometer (Rheinstetten, 
Germany) at $400 \mathrm{MHz}$ using TMS as internal standard and with residual signals of the deuterated solvent $\delta=7.26 \mathrm{ppm}$ for $\mathrm{CDCl}_{3}$ and $\delta 2.51 \mathrm{ppm}$ for DMSO- $d 6 .{ }^{13} \mathrm{C}$-NMR spectra were recorded on the same spectrometer at $100 \mathrm{MHz}$ and referenced to solvent signals $\delta=77 \mathrm{ppm}$ for $\mathrm{CDCl}_{3}$ and $\delta 39.50$ ppm for DMSO-d6. DEPT 135 NMR spectroscopy was used where appropriate to aid the assignment of signals in the ${ }^{1} \mathrm{H}$ and ${ }^{13} \mathrm{C}-\mathrm{NMR}$ spectra. The mass spectra were recorded on a Shimadzu GCMS-QP1000 EX mass spectrometer (Kyoto, Japan) at $70 \mathrm{eV}$ using the electron ionization technique. Homogeneity of all compounds synthesized was checked by TLC which was performed on Merck 60 (Munchen, Germany) ready-to-use silica gel plates to monitor the reactions and test the purity of the new synthesized compounds. Compounds $\mathbf{1 a}-\mathbf{c}$ were prepared by a previously reported method [2].

\subsection{General Procedure for the Preparation of Compounds $\mathbf{2 a - e}$}

An equimolar mixture of compounds $1 \mathbf{1 a}-\mathbf{c}(0.01 \mathrm{~mol})$ and the hydrazine derivatives, namely hydrazine hydrate, methyl hydrazine and phenyl hydrazine $(0.01 \mathrm{~mol})$ in $50 \mathrm{~mL}$ ethanol was refluxed for $6 \mathrm{~h}$. The solid that separated after cooling was filtered off, washed with petroleum ether (b.p $40-60{ }^{\circ} \mathrm{C}$ ), dried and then crystallized from ethanol.

3-Amino-5,5-di(4-methylphenyl)-1H-pyrazol-4(5H)-one (2a). Yield 73\%. m.p. 230-232 ${ }^{\circ} \mathrm{C}$. IR (KBr), $v \mathrm{~cm}^{-1}: 3310,3270\left(\mathrm{NH}_{2}, \mathrm{NH}\right), 3056\left(\mathrm{CH}_{\mathrm{Ar}}\right), 1713(\mathrm{CO}) .{ }^{1} \mathrm{H}-\mathrm{NMR}$ (DMSO-d6): $\delta$ at $2.13(\mathrm{~s}, 6 \mathrm{H}$, $\left.2 \mathrm{CH}_{3}\right), 7.09-7.86(\mathrm{~m}, 8 \mathrm{H}, \mathrm{ArH}), 8.83$ (bs, 2H, $\mathrm{NH}_{2}, \mathrm{D}_{2} \mathrm{O}$ exchangeable) $11.81\left(\mathrm{~s}, 1 \mathrm{H}, \mathrm{NH} \mathrm{D}_{2} \mathrm{O}\right.$ exchangeable), ${ }^{13} \mathrm{C}-\mathrm{NMR}\left(\mathrm{DMSO}-d_{6}\right) \delta \mathrm{ppm}: 23.6\left(\mathrm{CH}_{3}\right), 75.2(\mathrm{C} 5), 130.5,131.7,134.3,135.8,144.4$ (aromatic $+\mathrm{C}=\mathrm{N}), 191.6(\mathrm{C}=\mathrm{O})$. MS: $m / z 279\left[\mathrm{M}^{+}\right]$(34\%). Anal. Calc. for $\mathrm{C}_{17} \mathrm{H}_{17} \mathrm{~N}_{3} \mathrm{O}(279)$ : C, 73.11 ; H, 6.09; N 15.05; found: C, 73.39; H, 5.87; N, 15.44 .

3-Amino-5,5-di(4-methoxyphenyl)-1H-pyrazol-4(5H)-one (2b). Yield 77\%. m.p. 246-248 ${ }^{\circ} \mathrm{C}$. IR (KBr), $v \mathrm{~cm}^{-1}: 3436,3276\left(\mathrm{NH}_{2}, \mathrm{NH}\right), 3063\left(\mathrm{CH}_{\mathrm{Ar}}\right), 1712(\mathrm{CO}) .{ }^{1} \mathrm{H}-\mathrm{NMR}$ (DMSO-d6): $\delta 3.53\left(\mathrm{~s}, 6 \mathrm{H}, 2 \mathrm{OCH}_{3}\right)$, 7.43-7.82 (m, 8H, ArH), 12.12 (bs, $2 \mathrm{H}, \mathrm{NH}_{2}, \mathrm{D}_{2} \mathrm{O}$ exchangeable), and 13.24 (bs, $1 \mathrm{H}, \mathrm{NH}, \mathrm{D}_{2} \mathrm{O}$ exchangeable). ${ }^{13} \mathrm{C}-\mathrm{NMR}$ (DMSO- $\left.d_{6}\right) \delta$ ppm: $59.8\left(\mathrm{CH}_{3}\right), 77.1(\mathrm{C} 5), 116.7,128.4,134.6,143.8,153.5$ (aromatic $+\mathrm{C}=\mathrm{N}), 191.1(\mathrm{C}=\mathrm{O})$. MS: $m / z 311\left[\mathrm{M}^{+}\right]$(23.7\%). Anal. Calc. for $\mathrm{C}_{17} \mathrm{H}_{17} \mathrm{~N}_{3} \mathrm{O}_{3}(311)$ : $\mathrm{C}$, 65.59; H, 5.46; N, 15.43; found: C 65.23, H 5.31, N 15.05.

3-Amino-5,5-di(4-nitrophenyl)-1H-pyrazol-4(5H)-one (2c). Yield 65\%. m.p. 182-184 ${ }^{\circ} \mathrm{C}$. IR (KBr), $v \mathrm{~cm}^{-1}:$ 3457, $3338\left(\mathrm{NH}_{2}, \mathrm{NH}\right), 3065\left(\mathrm{CH}_{\mathrm{Ar}}\right), 1718(\mathrm{CO}) .{ }^{1} \mathrm{H}-\mathrm{NMR}\left(\mathrm{DMSO}-d_{6}\right): \delta$ 7.32-7.82 (m, 8H, $\mathrm{ArH}), 11.86$ (bs, 2H, NH $2, \mathrm{D}_{2} \mathrm{O}$ exchangeable) and 12.85 (bs, $1 \mathrm{H}, \mathrm{NH}, \mathrm{D}_{2} \mathrm{O}$ exchangeable). $\mathrm{MS}: m / z$ $341\left[\mathrm{M}^{+}\right]$(14.8\%). Anal. Calc. for $\mathrm{C}_{15} \mathrm{H}_{11} \mathrm{~N}_{5} \mathrm{O}_{5}$ (341): C, 52.78; H, 3.22; N, 20.53; found: C, 53.02; H, $3.08, \mathrm{~N}, 20.89$.

3-Amino-5,5-di(4-methylphenyl)-1-methyl-pyrazol-4(5H)-one (2d). Yield 64\%. m.p. 204-206 ${ }^{\circ} \mathrm{C}$. IR

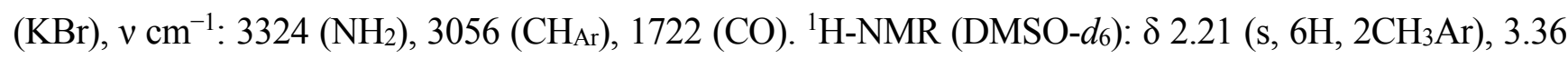
(s, 3H, CH3), 7.21-7.66 (m, 8H, ArH), 11.27 (bs, 2H, NH, $\mathrm{D}_{2} \mathrm{O}$ exchangeable). ${ }^{13} \mathrm{C}-\mathrm{NMR}$ (DMSO-d6) $\delta$ ppm: $22.7\left(\mathrm{CH}_{3}\right), 38.5\left(\mathrm{CH}_{3}\right), 78.6(\mathrm{C} 5), 130.6,132.3,133.5,137.9,144.1(\operatorname{aromatic}+\mathrm{C}=\mathrm{N}), 192.0$ $(\mathrm{C}=\mathrm{O})$. MS: $m / z 293\left[\mathrm{M}^{+}\right]$(12.9\%). Anal. Calc. for $\mathrm{C}_{18} \mathrm{H}_{19} \mathrm{~N}_{3} \mathrm{O}$ (293): C, 73.72; H, 6. 48; N, 14.33; found: C, 74.00; H, 6.75; N, 14.00 . 
3-Amino-5,5-di(4-methylphenyl)-1-phenyl-pyrazol-4(5H)-one (2e). Yield 60\%. m.p. 218-220 ${ }^{\circ} \mathrm{C}$. IR $(\mathrm{KBr}), v \mathrm{~cm}^{-1}: 3321\left(\mathrm{NH}_{2}\right), 3060\left(\mathrm{CH}_{\mathrm{Ar}}\right), 1720(\mathrm{CO}) .{ }^{1} \mathrm{HNMR}$ (DMSO-d6): $\delta 2.26(\mathrm{~s}, 6 \mathrm{H}$, $\left.2 \mathrm{CH}_{3}\right)$, multiplet at 7.28-7.80 (m, 13H, ArH), 10.17 (bs, $2 \mathrm{H}, \mathrm{NH}_{2}, \mathrm{D}_{2} \mathrm{O}$ exchangeable). ${ }^{13} \mathrm{C}-\mathrm{NMR}$ (DMSO-d6) $\delta$ ppm: $23.1\left(\mathrm{CH}_{3}\right), 76.7$ (C5), 117.8, 123.6, 126.8, 130.8, 132.1, 133.1, 135.5, 141.7, 144.6 (aromatic $+\mathrm{C}=\mathrm{N}), 192.6(\mathrm{C}=\mathrm{O})$. MS: $m / z 355\left[\mathrm{M}^{+}\right](8.9 \%)$. Anal. Calc. for $\mathrm{C}_{23} \mathrm{H}_{21} \mathrm{~N}_{3} \mathrm{O}(355)$ : $\mathrm{C}$, 77.74; H, 5.91; N, 11.83; found: C, 78.05; H, 5.77; N, 11.50 .

\subsection{General Procedure for the Preparation of the Compounds 3a-c}

A mixture of 1a-c $(0.01 \mathrm{~mol})$ and hydroxylamine hydrochloride $(1.03 \mathrm{~g} ; 0.015 \mathrm{~mol})$ in boiling pyridine $(50 \mathrm{~mL})$ was heated under reflux for $5 \mathrm{~h}$. The reaction mixture was allowed to cool, poured into ice $/ \mathrm{HCl}$. The product that precipitated was filtered, dried, and recrystallized from dioxane.

3-Amino-5,5-di(4-methylphenyl)isoxazol-4(5H)-one (3a). Yield 73\%. m.p. 222-224 ${ }^{\circ} \mathrm{C} . \mathrm{IR}(\mathrm{KBr})$, $v \mathrm{~cm}^{-1}: 3357\left(\mathrm{NH}_{2}\right), 3056\left(\mathrm{CH}_{\mathrm{Ar}}\right), 1706(\mathrm{CO}) .{ }^{1} \mathrm{H}-\mathrm{NMR}$ (DMSO-d $): \delta 2.11\left(\mathrm{~s}, 6 \mathrm{H}, 2 \mathrm{CH}_{3}\right), 7.25-7.80$ (m, 8H, Ar-H), 8.11 (bs, 2H, NH $2, \mathrm{D}_{2} \mathrm{O}$ exchangeable). ${ }^{13} \mathrm{C}-\mathrm{NMR}$ (DMSO-d6) $\delta$ ppm: $23.4\left(\mathrm{CH}_{3}\right)$, 101.7 (C5), 128.3, 130.4, 134.6, 140.0, 149.2 (aromatic $+\mathrm{C}=\mathrm{N}), 193.0(\mathrm{C}=\mathrm{O}) . \mathrm{MS}: m / z 280\left[\mathrm{M}^{+}\right.$] (4.6\%). Anal. Calc. for $\mathrm{C}_{17} \mathrm{H}_{16} \mathrm{~N}_{2} \mathrm{O}_{2}$ (280): C, 72.86; H, 5.71; N, 10.00; found: C, 72.57; H, 5.55; N, 10.32 .

3-Amino-5,5-di(4-methoxyphenyl)isoxazol-4(5H)-one (3b). Yield 77\%. m.p. 250-252 ${ }^{\circ} \mathrm{C}$. IR (KBr), $v \mathrm{~cm}^{-1}: 3299\left(\mathrm{NH}_{2}\right), 3054\left(\mathrm{CH}_{\mathrm{Ar}}\right), 1724$ (CO). ${ }^{1} \mathrm{H}-\mathrm{NMR}$ (DMSO-d6): $\delta 3.67$ (s, 6H, 2OCH $), 7.23-7.89$ (m, 8H, Ar-H), 9.21 (bs, 2H, NH $2, \mathrm{D}_{2} \mathrm{O}$ exchangeable). ${ }^{13} \mathrm{C}-\mathrm{NMR}$ (DMSO- $\left.d_{6}\right) \delta$ ppm: $58.2\left(\mathrm{CH}_{3}\right)$, 102.2 (C5), 120.6, 129.1, 135.2, 149.5, $154.4($ aromatic $+\mathrm{C}=\mathrm{N}), 192.1(\mathrm{C}=\mathrm{O}) . \mathrm{MS}: \mathrm{m} / z 312\left[\mathrm{M}^{+}\right.$] (15.7\%). Anal. Calc. for $\mathrm{C}_{17} \mathrm{H}_{16} \mathrm{~N}_{2} \mathrm{O}_{4}$ (312): C, 65.38; H, 5.13; N, 8.97; found: C, 65.00; H, 4.99; N 8.65.

3-Amino-5,5-di(4-nitrophenyl)isoxazol-4(5H)-one (3c). Yield 68\%. m.p. $204-206{ }^{\circ} \mathrm{C}$. IR (KBr), $v \mathrm{~cm}^{-1}$ : $3448\left(\mathrm{NH}_{2}\right), 3058$ (CHAr), 1723 (CO). ${ }^{1} \mathrm{H}-\mathrm{NMR}$ (DMSO-d6): $\delta$ 7.16-7.74 (m, 8H, Ar-H), 8.78 (bs, 2H, $\mathrm{NH}_{2}, \mathrm{D}_{2} \mathrm{O}$ exchangeable). MS: $m / z 342\left[\mathrm{M}^{+}\right]$(12.8\%). Anal. Calc. for $\mathrm{C}_{15} \mathrm{H}_{10} \mathrm{~N}_{4} \mathrm{O}_{6}(342)$ : C, 52.63; $\mathrm{H}$, 2.92 ; N, 16.37; found: C, 52.99; H, 2.80; N, 16.05 .

\subsection{6-Amino-7-hydroxy-7,7a-di(4-methylphenyl)-7,7a-dihydro-1H-pyrrolo[2,3-e][1,2,4]-triazine- 3(2H)-thione (4)}

An equimolar mixture of compound $1 \mathrm{a}(2.74 \mathrm{~g} ; 0.01 \mathrm{~mol})$ and thiosemicarbazide $(0.91 \mathrm{~g}, 0.01 \mathrm{~mol})$ in $50 \mathrm{~mL}$ ethanol was refluxed for $4 \mathrm{~h}$. The solid that separated after cooling was filtered off, dried and then, recrystallized from ethanol. Yield 70\%. m.p. 192-194 ${ }^{\circ} \mathrm{C}$. IR (KBr), $v \mathrm{~cm}^{-1}: 3543(\mathrm{OH}), 3408$ $\left(\mathrm{NH}_{2}\right), 3287,3209(\mathrm{NH}), 3052\left(\mathrm{CH}_{\mathrm{Ar}}\right), 2860(\mathrm{SH}), 1629(\mathrm{C}=\mathrm{N}) .{ }^{1} \mathrm{H}-\mathrm{NMR}$ (DMSO-d $)$ ): $\delta 2.19(\mathrm{~s}, 6 \mathrm{H}$, $\left.2 \mathrm{CH}_{3}\right), 5.63$ (bs, $1 \mathrm{H}, \mathrm{OH}, \mathrm{D}_{2} \mathrm{O}$ exchangeable), 7.16-7.72 (m, 8H, ArH) 8.77 (bs, 2H, $\mathrm{NH}_{2}, \mathrm{D}_{2} \mathrm{O}$ exchangeable) 12.07, 12.52 (bs, $2 \mathrm{H}, 2 \mathrm{NH}, \mathrm{D}_{2} \mathrm{O}$ exchangeable). ${ }^{13} \mathrm{C}-\mathrm{NMR}$ (DMSO- $d_{6}$ ) $\delta$ ppm: 23.1 $\left(\mathrm{CH}_{3}\right), 66.9,92.4$ (saturated-C), 124.7, 125.4, 126.4, 127.8, 135.3, 136.1, 140.1, 145.3, 157.2, 158.3 (Aromatic $+\mathrm{C}=\mathrm{N}), 179.9(\mathrm{C}=\mathrm{S})$. MS: $m / z 365\left[\mathrm{M}^{+}\right](21.4 \%)$. Anal. Calc. for $\mathrm{C}_{19} \mathrm{H}_{19} \mathrm{~N}_{5} \mathrm{OS}(365)$ : C 62.47, H 5.20, N 19.18, S 8.77; found: C 62.18, H 5.40, N 18.87, S 8.45. 
A mixture of 1a $(2.74 \mathrm{~g}, 0.01 \mathrm{~mol})$ and 2-amino-5-phenyl-1,3,4-thiadiazole $(1.77 \mathrm{~g} ; 0.01 \mathrm{~mol})$ in ethanol $(50 \mathrm{~mL})$ was refluxed for $4 \mathrm{~h}$, then left to cool. The separated solid product was filtered off, dried and recrystallized from toluene-ethanol mixture. Yield $65 \%$. m.p. $152-154{ }^{\circ} \mathrm{C}$. IR $(\mathrm{KBr}), v \mathrm{~cm}^{-1}$ : $3052\left(\mathrm{CH}_{\mathrm{Ar}}\right), 1668,1687(\mathrm{CO}), 1613(\mathrm{C}=\mathrm{N}) .{ }^{1} \mathrm{H}-\mathrm{NMR}$ (DMSO-d $)$ : $\delta 2.14$ (s, 3H, CH$), 7.24-7.81(\mathrm{~m}$, 13H, ArH). ${ }^{13} \mathrm{C}-\mathrm{NMR}$ (DMSO- $\left.d_{6}\right) \delta$ ppm: $22.4\left(\mathrm{CH}_{3}\right), 84.4$ (saturated-C), 127.3, 128.1, 129.6, 130.4, 131.7, 132.6, 133.4, 134. 3,135.2, 136.3, 137.5, 138.2, 141.7, 146.9, 149.3 (aromatic $+\mathrm{C}=\mathrm{N}), 175.8$, 199.1, (C=O). MS: $m / z 425\left[\mathrm{M}^{+}\right]$(5.1\%). Anal. Calc. for $\mathrm{C}_{25} \mathrm{H}_{19} \mathrm{~N}_{3} \mathrm{O}_{2} \mathrm{~S}(425): \mathrm{C}, 70.59 ; \mathrm{H}, 4.47 ; \mathrm{N}$, 9.88; S, 7.53; found: C, 70.95; H, 4.28; N, 9.52; S, 7.80.

\subsection{General Procedure for the Preparation of Compounds 6a-c}

An equimolar mixture of compounds $\mathbf{1 a}-\mathbf{c}(0.01 \mathrm{~mol})$ and hydrazine hydrate $(0.5 \mathrm{~mL}, 0.01 \mathrm{~mol})$ in $n$-butanol $(30 \mathrm{~mL})$ was refluxed for $8 \mathrm{~h}$. The solid that separated after cooling was filtered off, washed by petroleum ether (b.p. $40-60{ }^{\circ} \mathrm{C}$ ), dried and then crystallized from $n$-butanol to afford compounds 7a-c. The mother liquor ( $n$-butanol) was evaporated under vacuum till dryness. The obtained solid was crystallized from ethanol producing compounds $\mathbf{2} \mathbf{a}-\mathbf{c}$.

2,2,6,6-Tetra(4-methylphenyl)-5,6-dihydro-2H-pyrazolo[1,5-a]imidazole-3,7-dione (6a). Yield 30\%. m.p. 296-298 ${ }^{\circ} \mathrm{C}$. IR (KBr), $v \mathrm{~cm}^{-1}: 3119(\mathrm{NH}), 3074\left(\mathrm{CH}_{\mathrm{Ar}}\right), 1669,1652$ (C=O). ${ }^{1} \mathrm{H}-\mathrm{NMR}$ (DMSO-d6): $\delta$ 2.14, $2.36\left(\mathrm{~s}, 12 \mathrm{H}, 4 \mathrm{CH}_{3}\right), 7.04-7.58(\mathrm{~m}, 16 \mathrm{H}, \mathrm{ArH}), 12.33$ (bs, $1 \mathrm{H}, \mathrm{NH}, \mathrm{D}_{2} \mathrm{O}$ exchangeable). ${ }^{13} \mathrm{C}-$ NMR (DMSO-d6) $\delta$ ppm: $22.4\left(\mathrm{CH}_{3}\right), 79.9,87.8$ (saturated-C), 130.3, 131.4, 134.8, 137.1, 138.2, 140.1, 140.9, 148.8 (aromatic $+\mathrm{C}=\mathrm{N}), 184.5,190.8(\mathrm{C}=\mathrm{O})$. MS: $m / z 499\left[\mathrm{M}^{+}\right.$] $(7.75)$. Anal. Calc. for $\mathrm{C}_{33} \mathrm{H}_{29} \mathrm{~N}_{3} \mathrm{O}_{2}$ (499): C, 79.36; H, 5.81; N, 8.42; found: C, 79.32; H, 5.83; N, 8.45.

2,2,6,6-Tetra(4-methoxyphenyl)-5,6-dihydro-2H-pyrazolo[1,5-a]imidazole-3,7-dione (6b). Yield 27\%. m.p. 282-284 ${ }^{\circ} \mathrm{C}$. IR (KBr), $v \mathrm{~cm}^{-1}$ : $3331(\mathrm{NH}), 3050\left(\mathrm{CH}_{\mathrm{Ar}}\right), 1680,1666(\mathrm{C}=\mathrm{O}) .{ }^{1} \mathrm{H}-\mathrm{NMR}$ (DMSO- $\left.d 6\right)$ : $\delta 3.72,3.86\left(\mathrm{~s}, 12 \mathrm{H}, 4 \mathrm{OCH}_{3}\right), 7.54-8.20(\mathrm{~m}, 16 \mathrm{H}, \mathrm{ArH}), 12.51$ (bs, $1 \mathrm{H}, \mathrm{NH}, \mathrm{D}_{2} \mathrm{O}$ exchangeable). ${ }^{13} \mathrm{C}-\mathrm{NMR}$ (DMSO- $\left.d_{6}\right) \delta$ ppm: $57.1\left(\mathrm{CH}_{3}\right), 78.8,88.1$ (saturated-C), 118.7, 130.5, 133.2, 134.1, 146.3, 149.9 (aromatic $+\mathrm{C}=\mathrm{N}), 177.8,194.4(\mathrm{C}=\mathrm{O}) . \mathrm{MS}: \mathrm{m} / z 563\left[\mathrm{M}^{+}\right](13.4 \%)$. Anal. Calc. for $\mathrm{C}_{33} \mathrm{H}_{29} \mathrm{~N}_{3} \mathrm{O}_{6}$ (563): C, 70.34; H, 5.15; N, 7.46; found: C, 70.08; H, 4.93; N, 7.09.

2,2,6,6-Tetra(4-nitrophenyl)-5,6-dihydro-2H-pyrazolo[1,5-a]imidazole-3,7-dione (6c). Yield 25\%. m.p. 260-262 ${ }^{\circ} \mathrm{C}$. IR (KBr), $v \mathrm{~cm}^{-1}$ : $3276(\mathrm{NH}), 3073\left(\mathrm{CH}_{\mathrm{Ar}}\right), 1685,1669$ (C=O). ${ }^{1} \mathrm{H}-\mathrm{NMR}$ (DMSO-d6): $\delta$ 7.37-7.94 (m, 16H, ArH), 12.89 (bs, 1H, NH, D $2 \mathrm{O}$ exchangeable). MS: $m / z 623\left[\mathrm{M}^{+}\right.$] $(44.4 \%)$. Anal. Calc. for $\mathrm{C}_{29} \mathrm{H}_{17} \mathrm{~N}_{7} \mathrm{O}_{10}$ (623): C, 55.85; H, 2.72; N, 15.73; found: C, 55.84; H, 2.70; N, 15.70 .

\subsection{General Procedure for the Preparation of the Schiff's Bases 7a-d}

A mixture of compounds $\mathbf{2 a}-\mathbf{c}(0.01 \mathrm{~mol})$ and aromatic aldehyde, namely benzaldehyde and/or $p$-chlorobenzaldehyde $(0.01 \mathrm{~mol})$ in ethanol $(50 \mathrm{~mL})$ was refluxed for $4 \mathrm{~h}$. The solid that separated after cooling was filtered off, dried and then crystallized from ethanol. 
3-(Benzylideneamino)-5,5-di(4-methylphenyl)-1H-pyrazol-4(5H)-one (7a). Yield 70\%. m.p. 212-214 ${ }^{\circ} \mathrm{C}$. IR (KBr), $v \mathrm{~cm}^{-1}: 3221(\mathrm{NH}), 3056$ (CHAr), $1676(\mathrm{CO}) .{ }^{1} \mathrm{H}-\mathrm{NMR}$ (DMSO-d6): $\delta 2.25$ (s, 6H, 2CH3), $6.22(\mathrm{~s}, 1 \mathrm{H}, \mathrm{CH}=), 7.19-7.92(\mathrm{~m}, 13 \mathrm{H}, \mathrm{ArH}), 11.24$ (bs, $1 \mathrm{H}, \mathrm{NH}, \mathrm{D}_{2} \mathrm{O}$ exchangeable). ${ }^{13} \mathrm{C}-\mathrm{NMR}$ (DMSO-d6) $\delta$ ppm: $23.3\left(\mathrm{CH}_{3}\right), 75.7$ (saturated-C), 127.4, 128.6, 130.2, 131.4, 133.9, 134.7, 135.6, 137.9, 147.1, $152.6($ aromatic $+\mathrm{C}=\mathrm{N}), 191.5(\mathrm{C}=\mathrm{O})$. MS: $m / z 367\left[\mathrm{M}^{+}\right]$, 274, 194, 178, 166, 145. Anal. Calc. for $\mathrm{C}_{24} \mathrm{H}_{21} \mathrm{~N}_{3} \mathrm{O}$ (367): C, 78.47; H, 5.72; N, 11.44; found: C, 78.81; H, 5.55; N 11.12.

3-(Benzylideneamino)-5,5-di(4-methoxyphenyl)-1H-pyrazol-4(5H)-one (7b). Yield 75\%. m.p. 226-228 ${ }^{\circ} \mathrm{C}$. IR (KBr), $v \mathrm{~cm}^{-1}: 3211(\mathrm{NH}), 3058\left(\mathrm{CH}_{\mathrm{Ar}}\right), 1670(\mathrm{CO}) .{ }^{1} \mathrm{H}-\mathrm{NMR}\left(\mathrm{DMSO}-d_{6}\right): \delta 3.71\left(\mathrm{~s}, 6 \mathrm{H}, 2 \mathrm{OCH}_{3}\right)$, $6.31(\mathrm{~s}, 1 \mathrm{H}, \mathrm{CH}=), 7.16-7.97(\mathrm{~m}, 13 \mathrm{H}, \mathrm{ArH}), 11.32$ (bs, 1H, NH, $\mathrm{D}_{2} \mathrm{O}$ exchangeable). ${ }^{13} \mathrm{C}-\mathrm{NMR}$ (DMSO-d6) $\delta$ ppm: $59.3\left(\mathrm{CH}_{3}\right), 77.4$ (saturated-C), 116.2, 128.6, 130.1, 131.5, 133.2, 134.6, 135.8, 148.5, 149.8 153.5(aromatic $+\mathrm{C}=\mathrm{N}), 189.5(\mathrm{C}=\mathrm{O})$. MS: $m / z 399\left[\mathrm{M}^{+}\right](28.2 \%)$. Anal. Calc. for $\mathrm{C}_{24} \mathrm{H}_{21} \mathrm{~N}_{3} \mathrm{O}_{3}$ (399): C, 72.18; H, 5.26; N, 10.53; found: C, 71.83; H, 5.61; N, 10.35.

3-(Benzylideneamino)-5,5-di(4-nitrophenyl)-1H-pyrazol-4(5H)-one (7c). Yield 65\%. m.p. 210-212 ${ }^{\circ} \mathrm{C}$. IR (KBr), $v \mathrm{~cm}^{-1}: 3278(\mathrm{NH}), 3080\left(\mathrm{CH}_{\mathrm{Ar}}\right), 1680(\mathrm{CO}) .{ }^{1} \mathrm{H}-\mathrm{NMR}$ (DMSO-d6): $\delta 6.47$ (s, 1H, CH=), 7.14-8.00 (m, 13H, ArH), 10.11 (bs, $1 \mathrm{H}, \mathrm{NH}, \mathrm{D}_{2} \mathrm{O}$ exchangeable). MS: $m / z 429$ [M ${ }^{+}$] (25.6\%). Anal. Calc. for $\mathrm{C}_{22} \mathrm{H}_{15} \mathrm{~N}_{5} \mathrm{O}_{5}$ (429): C, 67.71; H, 4.07; N, 13.16; found: C, 67.70; H, 4.02; N, 13.15 .

3-(4-Chlorobenzylidenimino)-5,5-di(4-methylphenyl)-1H-pyrazol-4(5H)-one (7d). Yield 72\%. m.p. 226-228 ${ }^{\circ} \mathrm{C}$. IR (KBr), $v \mathrm{~cm}^{-1}: 3290(\mathrm{NH}), 3052\left(\mathrm{CH}_{\mathrm{Ar}}\right), 1672(\mathrm{CO}) .{ }^{1} \mathrm{H}-\mathrm{NMR}$ (DMSO-d6): $\delta 2.28$ (s, $\left.6 \mathrm{H}, 2 \mathrm{CH}_{3}\right), 6.57$ (s, 1H, CH=), 7.31-7.95 (m, 12H, ArH), 11.00 (bs, 1H, NH, $\mathrm{D}_{2} \mathrm{O}$ exchangeable). ${ }^{13} \mathrm{C}-\mathrm{NMR}$ (DMSO- $\left.d_{6}\right) \delta$ ppm: $23.0\left(\mathrm{CH}_{3}\right), 75.1$ (saturated-C), 127.7, 128.8, 130.0, 131.6, 133.5, 134.8, 135.8, 137.5, 147.4, 153.1 (aromatic $+\mathrm{C}=\mathrm{N}), 191.8(\mathrm{C}=\mathrm{O}) . \mathrm{MS}: m / z 401\left[\mathrm{M}^{+}\right](33.6 \%),[\mathrm{M}+2]$ (11.1\%). Anal. Calc. for $\mathrm{C}_{24} \mathrm{H}_{20} \mathrm{~N}_{3} \mathrm{OCl}$ (401.5): C, 71.73; H, 4.98; N, 10.46; Cl, 8.84; found: C, 70.65; $\mathrm{H}, 4.26 ; \mathrm{N}, 11.21 ; \mathrm{Cl}, 8.51$.

\subsection{3,3-Di(4-methylphenyl)-2,3-dihydro-1H-pyrazolo[4,3-b]pyrazin-6(7H)-one (8)}

A mixture of compound 2a (2.76 g; $0.01 \mathrm{~mol})$ and methyl glycinate $(1 \mathrm{~mL}, 0.01 \mathrm{~mol})$ in ethanol $(50 \mathrm{~mL})$ was refluxed for $4 \mathrm{~h}$. The solid that separated out after cooling was filtered off, dried and then recrystallized from dioxane. Yield 70\%. m.p. $164-166{ }^{\circ} \mathrm{C}$. IR $(\mathrm{KBr}), v \mathrm{~cm}^{-1}: 3277-3226(\mathrm{NH}), 3050$ $\left(\mathrm{CH}_{\mathrm{Ar}}\right), 1668(\mathrm{C}=\mathrm{O}) .{ }^{1} \mathrm{H}-\mathrm{NMR}$ (DMSO-d6): $\delta 2.20$ (s, 6H, 2CH3), 6.77 (s, 1H, CHpy), 7.21-7.80 (m, $8 \mathrm{H}, \mathrm{ArH}), 6.4,9.7$ and 12.4 (3bs, $3 \mathrm{H}, 3 \mathrm{NH}, \mathrm{D}_{2} \mathrm{O}$ exchangeable). ${ }^{13} \mathrm{C}-\mathrm{NMR}$ (DMSO- $\left.d_{6}\right) \delta$ ppm: 23.0 $\left(\mathrm{CH}_{3}\right), 75.9$ (saturated-C), 115.8, 130.1, 131.5, 134.0, 137.4, 138.7, 142.6 (aromatic $\left.+\mathrm{C}=\mathrm{N}\right), 178.4$ $(\mathrm{C}=\mathrm{O})$. MS: $m / z 318\left[\mathrm{M}^{+}\right]$(23.9\%). Anal. Calc. for $\mathrm{C}_{19} \mathrm{H}_{18} \mathrm{~N}_{4} \mathrm{O}$ (318): C, 71.70; H, 5.66; N, 17.61; found: C, 71.47; H, 5.48; N, 17.28.

\subsection{General Procedure for the Preparation of the Compounds $9 \mathbf{9}-\mathbf{c}$}

A mixture of compounds $7 \mathbf{a}-\mathbf{c}(0.01 \mathrm{~mol})$ and ammonium acetate $(2.31 \mathrm{~g}, 0.03 \mathrm{~mol})$ was heated in an oil bath at $90{ }^{\circ} \mathrm{C}$ for $3 \mathrm{~h}$. The mixture was left to cool then washed with water several times. The solid product was filtered off, dried and then, crystallized from dioxane. 
3,3-Di(4-methylphenyl)-5-phenyl-4,5-dihydroimidazo[4,5-c]pyrazole (9a). Yield 69\%. m.p. 164-166 ${ }^{\circ} \mathrm{C}$. IR (KBr), $v \mathrm{~cm}^{-1}:$ 3233, $3196(\mathrm{NH}), 3066\left(\mathrm{CH}_{\mathrm{Ar}}\right), 1604(\mathrm{C}=\mathrm{N}) .{ }^{1} \mathrm{H}-\mathrm{NMR}$ (DMSO-d6): $\delta 2.26(\mathrm{~s}, 6 \mathrm{H}$, $\left.2 \mathrm{CH}_{3}\right), 5.13$ (s, 1H, imidazol), 7.23-7.73 (m, 13H, ArH), 9.71 and 10.49 (bs, 2H, 2NH, D $2 \mathrm{O}$ exchangeable). ${ }^{13} \mathrm{C}-\mathrm{NMR}$ (DMSO- $\left.d_{6}\right) \delta$ ppm: $22.9\left(\mathrm{CH}_{3}\right), 67.1,75.8$ (saturated-C), 117.6, 124.5, 127.8, 128.7, 130.4, 131.9, 133.8, 137.7, 141.4, 143.3 (aromaic-C). MS: $m / z 366$ [M ${ }^{+}$] (55.1\%). Anal. Calc. for $\mathrm{C}_{24} \mathrm{H}_{22} \mathrm{~N}_{4}$ (366): C, 78.69; H, 6.01; N, 15.30; found: C, 78.96; H, 5.75; N, 15.62.

3,3-Di(4-methoxyphenyl)-5-phenyl-4,5-dihydroimidazo[4,5-c]pyrazole (9b). Yield 74\%. m.p. 180-182 ${ }^{\circ} \mathrm{C}$. IR (KBr), $v \mathrm{~cm}^{-1}$ : 3280, $3208(\mathrm{NH}), 3050\left(\mathrm{CH}_{\mathrm{Ar}}\right), 1614(\mathrm{C}=\mathrm{N}) .{ }^{1} \mathrm{H}-\mathrm{NMR}\left(\mathrm{DMSO}-d_{6}\right): \delta 3.85(\mathrm{~s}, 6 \mathrm{H}$, $\left.2 \mathrm{CH}_{3}\right), 5.28(\mathrm{~s}, 1 \mathrm{H}$, imidazol), 7.12-7.92 (m, 13H, $\mathrm{ArH}), 8.88$ and $9.99\left(\mathrm{bs}, 2 \mathrm{H}, 2 \mathrm{NH}, \mathrm{D}_{2} \mathrm{O}\right.$ exchangeable). ${ }^{13} \mathrm{C}-\mathrm{NMR}$ (DMSO- $\left.d_{6}\right) \delta$ ppm: $58.7\left(\mathrm{CH}_{3}\right), 67.4,75.2$ (saturated-C), 118.5, 124.8, 127.2, 128.9, 130.7, 131.7, 133.5, 136.7, 145.8, 149.6 (aromatic-C). MS: $m / z 398$ [M $\left.{ }^{+}\right]$(43.8\%). Anal. Calc. for $\mathrm{C}_{24} \mathrm{H}_{22} \mathrm{~N}_{4} \mathrm{O}_{2}$ (398): C, 72.36; H, 5.53; N, 14.07; found: C, 71.98; H, 5.75; N, 13.76.

3,3-Di(4-nitrophenyl)-5-phenyl-4,5-dihydroimidazo[4,5-c]pyrazole (9c). Yield 66\%. m.p. 224-228 ${ }^{\circ} \mathrm{C}$. IR (KBr), $v \mathrm{~cm}^{-1}: 3278,3202(\mathrm{NH}), 3080\left(\mathrm{CH}_{\mathrm{Ar}}\right), 1629(\mathrm{C}=\mathrm{N}) .{ }^{1} \mathrm{H}-\mathrm{NMR}\left(\mathrm{DMSO}-d_{6}\right): \delta 5.41(\mathrm{~s}, 1 \mathrm{H}$, imidazol), 7.34-8.23 (m, 13H, ArH), 10.22 and 11.09 (bs, 2H, 2NH, $\mathrm{D}_{2} \mathrm{O}$ exchangeable). ${ }^{13} \mathrm{C}-\mathrm{NMR}$ (DMSO-d6) $\delta$ ppm: 66.8, 75.2 (saturated-C) 120.8, 124.2, 126.1, 127.8, 129.5, 130.6, 131.7, 142.5, 146.3, 147.8 (aromatic-C). MS: $m / z 428$ [ $\mathrm{M}^{+}$] (48.2\%). Anal. Calc. for $\mathrm{C}_{22} \mathrm{H}_{16} \mathrm{~N}_{6} \mathrm{O}_{4}$ (428): C, 61.68; $\mathrm{H}$, $3.74 ; \mathrm{N}, 19.63$; found: C, 61.89; H, 4.01; N, 19.99 .

\subsection{General Procedure for the Preparation of the Compounds 10a-c}

An equimolar mixture of compounds $7 \mathbf{a}-\mathbf{c}(0.01 \mathrm{~mol})$ and hydrazine hydrate $(0.5 \mathrm{~mL}, 0.01 \mathrm{~mol})$ in ethanol $(50 \mathrm{~mL})$ was refluxed for $6 \mathrm{~h}$. The solid that separated out after cooling was filtered off, washed with petroleum ether (b.p. $40-60^{\circ} \mathrm{C}$ ), dried and then crystallized from ethanol.

7,7-Di(4-methylphenyl)-3-phenyl-2,3,4,7-tetrahydro-1H-pyrazolo[3,4-e][1,2,4]triazine (10a). Yield 70\%. m.p. ${ }^{190-192}{ }^{\circ} \mathrm{C}$. IR (KBr), $v \mathrm{~cm}^{-1}$ : 3298, 3260, $3182(\mathrm{NH}), 3063\left(\mathrm{CH}_{\mathrm{Ar}}\right), 1630(\mathrm{C}=\mathrm{N}) .{ }^{1} \mathrm{H}-\mathrm{NMR}$ (DMSO-d6): $\delta 2.22\left(\mathrm{~s}, 6 \mathrm{H}, 2 \mathrm{CH}_{3}\right), 5.55(\mathrm{~s}, 1 \mathrm{H}$, triazine), 7.13-7.88 (m, 13H, ArH), 9.13, 10.27 and 11.07 (bs, 3H, NH, D $2 \mathrm{O}$ exchangeable). ${ }^{13} \mathrm{C}-\mathrm{NMR}$ (DMSO-d6) $\delta$ ppm: $22.5\left(\mathrm{CH}_{3}\right), 70.2,76.4$ (saturated-C), 118.2, 124.8, 127.4, 128.9, 130.5, 131.7, 133.5, 137.2, 142.1, 145.1 (aromatic-C). MS: $m / z 381\left[\mathrm{M}^{+}\right]$(22.3\%). Anal. Calc. for $\mathrm{C}_{24} \mathrm{H}_{23} \mathrm{~N}_{5}$ (381): C, 75.59; H, 6.04; N, 18.37; found: C, 75.75; $\mathrm{H}, 5.86 ; \mathrm{N}, 18.02$.

7,7-Di(4-methoxyphenyl)-3-phenyl-2,3,4,7-tetrahydro-1H-pyrazolo[3,4-e][1,2,4]triazine (10b). Yield 72\%. m.p. $181-183{ }^{\circ} \mathrm{C}$. IR (KBr), $v \mathrm{~cm}^{-1}$ : 3284, 3222, $3200(\mathrm{NH}), 3056\left(\mathrm{CH}_{\mathrm{Ar}}\right), 1641(\mathrm{C}=\mathrm{N}) .{ }^{1} \mathrm{H}-\mathrm{NMR}$ (DMSO-d $\left.d_{6}\right): \delta 3.79\left(\mathrm{~s}, 6 \mathrm{H}, 2 \mathrm{OCH}_{3}\right), 5.42(\mathrm{~s}, 1 \mathrm{H}$, triazine), 7.30-7.79 (m, 13H, ArH), 9.38, 10.30 and 12.00 (bs, 3H, NH, D $2 \mathrm{O}$ exchangeable). ${ }^{13} \mathrm{C}-\mathrm{NMR}$ (DMSO-d6) $\delta \mathrm{ppm}: 59.1\left(\mathrm{CH}_{3}\right), 70.8,77.3$ (saturated-C), 117.8, 124.1, 126.8, 128.6, 129.9, 131.4, 133.8, 137.1, 146.2, 150.5 (aromatic-C). MS: $m / z 413\left[\mathrm{M}^{+}\right]$(26.1\%). Anal. Calc. for $\mathrm{C}_{24} \mathrm{H}_{23} \mathrm{~N}_{5} \mathrm{O}_{2}$ (413): C, 69.73; H, 5.57; N, 16.95; found: C, 70.01; $\mathrm{H}, 5.71 ; \mathrm{N}, 16.60$; 
m.p. 241-243 ${ }^{\circ} \mathrm{C}$. IR (KBr), $v \mathrm{~cm}^{-1}$ : v 3300, 3268, $3190(\mathrm{NH}), 3080\left(\mathrm{CH}_{\mathrm{Ar}}\right), 1632(\mathrm{C}=\mathrm{N}) .{ }^{1} \mathrm{H}-\mathrm{NMR}$ (DMSO-d6): $\delta 4.8$ (s, $1 \mathrm{H}$, triazine), 7.31-8.28 (m, 13H, ArH), 8.49, 10.01 and 11.77 (bs, 3H, NH, $\mathrm{D}_{2} \mathrm{O}$ exchangeable). ${ }^{13} \mathrm{C}-\mathrm{NMR}$ (DMSO- $\left.d_{6}\right) \delta \mathrm{ppm:} \mathrm{69.9,} 75.9$ (saturated-C) 116.4, 121.4, 126.5, 127.6, 129.7, 130.7, 132.1, 138.0, 146.0, 149.9 (aromatic-C). MS: $m / z 443\left[\mathrm{M}^{+}\right.$] (27.7\%). Anal. Calc. for $\mathrm{C}_{22} \mathrm{H}_{17} \mathrm{~N}_{7} \mathrm{O}_{4}$ (443): C, 59.59; H, 3.84; N, 22.12; found: C, 59.85; H, 4.07; N, 21.77.

\subsection{4,4-Di(4-methylphenyl)-3,4-dihydro-7H-2a,3,5,7a-tetraazacyclopenta[c,d]indene-1,7-(2H)-dione (11).}

An equimolar mixture of compound $8(3.18 \mathrm{~g} ; 0.01 \mathrm{~mol})$ and chloroacetic acid (1 g; $0.01 \mathrm{~mol})$ in phosphorous oxychloride $(20 \mathrm{~mL})$ was refluxed for $2 \mathrm{~h}$. After cooling, the reaction mixture was poured onto ice/ $\mathrm{H}_{2} \mathrm{O}$, the solid that separated out was filtered off, washed with petroleum ether (b.p. $40-60{ }^{\circ} \mathrm{C}$ ), dried and then crystallized from toluene. Yield 55\%. m.p. $170-172{ }^{\circ} \mathrm{C}$. IR (KBr), v cm ${ }^{-1}: 3222(\mathrm{NH})$, 1677, $1655(\mathrm{CO}), 1613(\mathrm{C}=\mathrm{N}) .{ }^{1} \mathrm{H}-\mathrm{NMR}\left(\mathrm{DMSO}-d_{6}\right): \delta 2.16\left(\mathrm{~s}, 6 \mathrm{H}, 2 \mathrm{CH}_{3}\right), 4.17\left(\mathrm{~s}, 2 \mathrm{H}, \mathrm{CH}_{2}\right), 6.72(\mathrm{~s}$, $1 \mathrm{H}$, pyrazine), 7.44-7.76 (m, 8H, ArH), 8.72 (bs, $1 \mathrm{H}, \mathrm{NH}, \mathrm{D}_{2} \mathrm{O}$ exchangeable). ${ }^{13} \mathrm{C}-\mathrm{NMR}$ (DMSO- $\left.d_{6}\right)$ $\delta$ ppm: $23.2\left(\mathrm{CH}_{3}\right), 57.1,76.8$ (sp3-C), 117.4, 127.8, 130.7, 131.9, 134.1, 137.6, 145.1 (aromatic + sp2-C), 160.2, $170.1(\mathrm{C}=\mathrm{O})$. MS: $m / z 358\left[\mathrm{M}^{+}\right]$(43.7\%). Anal. Calc. for $\mathrm{C}_{21} \mathrm{H}_{18} \mathrm{~N}_{4} \mathrm{O}_{2}$ (358): C, 70.39; H, 5.03; N, 15.64; found: C, 70.08; H, 4.81; N, 16.01 .

\subsection{0,10-Di(4-methylphenyl)-4,10-dihydro-3H,6H-pyrazolo[1',2':1,2]pyrazolo[3,4-b]-pyrazine- 3,6,8-(7H)-trione (12)}

A mixture of compound 8 (3.18 $\mathrm{g} ; 0.01 \mathrm{~mol})$ and diethyl malonate $(2.4 \mathrm{~mL} ; 0.015 \mathrm{~mol})$ in ethanol $(50 \mathrm{~mL})$ was refluxed for $4 \mathrm{~h}$. The solid that separated out after cooling was filtered off, washed with petroleum ether (b.p. $40-60{ }^{\circ} \mathrm{C}$ ), dried and then recrystallized from ethanol. Yield 57\%. m.p. $224-226^{\circ} \mathrm{C}$. IR (KBr), $v \mathrm{~cm}^{-1}: 3245(\mathrm{NH}), 1691,1672,1659(\mathrm{CO}), 1620(\mathrm{C}=\mathrm{N}),{ }^{1} \mathrm{H}-\mathrm{NMR}$ (DMSO-d6): $\delta 2.15$ (s, $\left.6 \mathrm{H}, 2 \mathrm{CH}_{3}\right), 4.51\left(\mathrm{~s}, 2 \mathrm{H}, \mathrm{COCH} \underline{\mathrm{H}}_{2} \mathrm{CO}\right), 6.81$ (s, 1H, pyrazine), 7.32-7.83 (s,8H, ArH), 10.14 (bs, 1H, NH, $\mathrm{D}_{2} \mathrm{O}$ exchangeable). ${ }^{13} \mathrm{C}-\mathrm{NMR}$ (DMSO- $\left.d_{6}\right) \delta \mathrm{ppm}: 23.0\left(\mathrm{CH}_{3}\right), 49.4,76.0$ (saturated-C), 117.0, 127.4, 130.0, 131.2, 134.7, 138.0, $145.9($ aromatic $+\mathrm{C}=\mathrm{N}), 161.3,170.9,175.3(\mathrm{C}=\mathrm{O}) . \mathrm{MS}: \mathrm{m} / z 386\left[\mathrm{M}^{+}\right]$ (44.4\%). Anal. Calc. for $\mathrm{C}_{22} \mathrm{H}_{18} \mathrm{~N}_{4} \mathrm{O}_{3}$ (386): C, 68.39; H, 4.66; N, 14.51; found: C, 68.07; H, 4.88; $\mathrm{N}, 14.22$.

\subsection{7-Benzylidene-10,10-di(4-methylphenyl)-4,10-dihydro-3H,6H-pyrazolo[1',2':1,2]-pyrazolo[3,4-} b]pyrazine-3,6,8(7H)-trione (13)

An equimolar mixture of compound 12 (3.86 g; $0.01 \mathrm{~mol})$ and benzaldehyde (1.06 mL; $0.01 \mathrm{~mol})$ in ethanol $(50 \mathrm{~mL})$ was refluxed for $6 \mathrm{~h}$. The solid that separated after cooling was filtered off, dried and then crystallized from $n$-butanol. Yield 24\%. m.p. $266-268^{\circ} \mathrm{C}$. IR (KBr), $v \mathrm{~cm}^{-1}: 3290(\mathrm{NH}), 1687$, 1677, 1666, (CO), $1626(\mathrm{C}=\mathrm{N}) .{ }^{1} \mathrm{H}-\mathrm{NMR}\left(\mathrm{DMSO}-d_{6}\right): \delta 2.26\left(\mathrm{~s}, 6 \mathrm{H}, 2 \mathrm{CH}_{3}\right), 6.44,6.87(\mathrm{bs}, 2 \mathrm{H}$, benzylic and pyrazine), 7.27-7.87 (m, 13H, ArH), 9.82 (bs, $1 \mathrm{H}, \mathrm{NH}, \mathrm{D}_{2} \mathrm{O}$ exchangeable). ${ }^{13} \mathrm{C}-\mathrm{NMR}$ (DMSO-d6)

$\delta$ ppm: $23.4\left(\mathrm{CH}_{3}\right), 76.5$ (saturated-C), 117.8, 125.1, 126. 4, 127.7, 128.9, 130.0, 131.2, 133.0, 134.7, 136.3, 138.0, 145.9, 150.7 (aromatic $+\mathrm{C}=\mathrm{N}), 160.5,171.2,173.7(\mathrm{C}=\mathrm{O}) . \mathrm{MS}: m / z 474\left[\mathrm{M}^{+}\right](77.6 \%)$. Anal. Calc. for $\mathrm{C}_{29} \mathrm{H}_{22} \mathrm{~N}_{4} \mathrm{O}_{3}$ (474): C, 73.42; H, 4.64; N, 11.81; found: C 73.05; H, 4.35; N, 12.13 . 


\subsection{General Procedure for the Preparation of Compounds $14 a, b$}

An equimolar mixture of compound $1 \mathrm{a}(2.74 \mathrm{~g} ; 0.01 \mathrm{~mol})$ and an active methylene precursor, e.g., acetylacetone and/or cyclopentanone $(0.01 \mathrm{~mol})$ and aqueous $\mathrm{NaOH}(50 \%, 8 \mathrm{~mL})$ in ethanol $(50 \mathrm{~mL})$ was refluxed for $3 \mathrm{~h}$ and left overnight. The reaction mixture was poured into ice/ $\mathrm{HCl}$. The solid so formed filtered off, washed with water, dried and then crystallized from ethanol.

3,3-Diacetyl-1,2-di(4-methylphenyl)-1,2-dicyanopropanol (14a). Yield 70\%. m.p. 220-222 ${ }^{\circ} \mathrm{C}$. IR $(\mathrm{KBr}), v \mathrm{~cm}^{-1}: 3380(\mathrm{OH}), 3062\left(\mathrm{CH}_{\mathrm{Ar}}\right), 2240,2220(\mathrm{CN}), 1688(\mathrm{CO}) .{ }^{1} \mathrm{H}-\mathrm{NMR}\left(\mathrm{DMSO}-d_{6}\right): \delta 2.19$ (s, $\left.6 \mathrm{H}, 2 \mathrm{CH}_{3}\right), 2.43\left(\mathrm{~s}, 6 \mathrm{H}, 2 \mathrm{CH}_{3}\right), 4.29(\mathrm{~s}, 1 \mathrm{H}, \mathrm{COCHCO}), 5.55$ (bs, $1 \mathrm{H}, \mathrm{OH}, \mathrm{D}_{2} \mathrm{O}$ exchangeable), 7.25$7.84(\mathrm{~m}, 8 \mathrm{H}, \mathrm{ArH}) .{ }^{13} \mathrm{C}-\mathrm{NMR}$ (DMSO- $\left.d_{6}\right) \delta \mathrm{ppm}: 22.5,30.2,\left(\mathrm{CH}_{3}\right), 36.0,63.1,78.2$ (saturated-C), 116.8, $120.0(\mathrm{CN}), 125.3,127.1,129.6,131.2,134.6,136.8,141.1,146.9$ (aromatic-C), $200.2(\mathrm{C}=\mathrm{O})$. MS: $m / z 374\left[\mathrm{M}^{+}\right]$(87.4\%). Anal. Calc. for $\mathrm{C}_{23} \mathrm{H}_{22} \mathrm{~N}_{2} \mathrm{O}_{3}$ (374): C, 73.80; H, 5.88; N, 7.48; found: C, 74.15; H, 5.67; N, 7.16.

1,2-Dicyano-1,2-di(4-methylphenyl)-2-(2-oxocyclopentyl)ethanol (14b). Yield 67\%. m.p. 192-194 ${ }^{\circ} \mathrm{C}$. IR (KBr), $v \mathrm{~cm}^{-1}: 3367(\mathrm{OH}), 3051\left(\mathrm{CH}_{\mathrm{Ar}}\right), 2950\left(\mathrm{CH}_{\mathrm{Ali}}\right), 2243,2221(\mathrm{CN}), 1677$ (CO), ${ }^{1} \mathrm{H}-\mathrm{NMR}$ (DMSO-d6): $\delta$ 1.79-2.01 (m, 6H, CHcyclopent.), 2.13 (s, 6H, 2CH3), 2.31 (s, 1H, CHcyclopent), 5.88 (bs, $1 \mathrm{H}, \mathrm{OH}, \mathrm{D}_{2} \mathrm{O}$ exchangeable), $7.15-7.78$ (m, 8H, ArH). ${ }^{13} \mathrm{C}-\mathrm{NMR}$ (DMSO- $\left.d_{6}\right) \delta$ ppm: 15.4, 21.5, 22.7, 36.9, 42.3, 52.0, 77.2 (saturated-C), 116.1, 119.2 (CN), 125.5, 127.4, 129.9, 130.9, 133.8, 136.3, 141.1, 145.2 (aromatic-C), $209.0(\mathrm{C}=\mathrm{O})$. MS: $m / z 358\left[\mathrm{M}^{+}\right]$(77.7\%). Anal. Calc. for $\mathrm{C}_{23} \mathrm{H}_{22} \mathrm{~N}_{2} \mathrm{O}_{2}$ (358): C, 76.09; H, 6.15; N 7.82; found: C, 75.73; H, 5.89; N, 8.14.

\subsection{General Procedure for the Preparation of the Compounds 15a,b}

Compounds 14a,b $(0.01 \mathrm{~mol})$ and ammonium acetate $(2.31 \mathrm{~g}, 0.03 \mathrm{~mol})$ were mixed thoroughly and fused in an oil bath at $150^{\circ} \mathrm{C}$ for $2 \mathrm{~h}$. Left to cool, then washed with water several times. The solid product was dried and recrystallized from benzene.

5-Acetyl-2-amino-6-methyl-4,4-di(4-methylpheny)pyridin-3(4H)-one (15a). Yield 74\%. m.p. 210-212 ${ }^{\circ} \mathrm{C}$. IR (KBr), $v \mathrm{~cm}^{-1}: 3285\left(\mathrm{NH}_{2}\right) .1691,1665(\mathrm{CO}), 1630(\mathrm{C}=\mathrm{N}),{ }^{1} \mathrm{H}-\mathrm{NMR}\left(\mathrm{DMSO}-d_{6}\right): \delta 2.14(\mathrm{~s}, 6 \mathrm{H}$, $\left.2 \mathrm{CH}_{3}\right), 2.41\left(\mathrm{~s}, 3 \mathrm{H}, \mathrm{CH}_{3}\right), 2.77\left(\mathrm{~s}, 3 \mathrm{H}, \mathrm{CH}_{3}\right), 7.44-7.83(\mathrm{~m}, 8 \mathrm{H}, \mathrm{ArH}), 9.42\left(\mathrm{bs}, 2 \mathrm{H}, \mathrm{NH}_{2}, \mathrm{D}_{2} \mathrm{O}\right.$ exchangeable). ${ }^{13} \mathrm{C}-\mathrm{NMR}$ (DMSO- $\left.d_{6}\right) \delta$ ppm: 22.5, 23.6, $28.5\left(\mathrm{CH}_{3}\right), 52.0$ (saturated-C), 128.7, 130.0, 131.9, 133.2, 137.0, 140.9, $145.6($ aromatic $+\mathrm{C}=\mathrm{N}), 192.7,198.00(\mathrm{C}=\mathrm{O}) . \mathrm{MS}: \mathrm{m} / z 346\left[\mathrm{M}^{+}\right](41.8 \%)$. Anal. Calc. for $\mathrm{C}_{22} \mathrm{H}_{22} \mathrm{~N}_{2} \mathrm{O}_{2}$ (346): C, 76.30; H, 6.36; N, 8.09; found: C, 76.60; H, 6.00; N, 8.41.

2-Amino-4,4-(4-methylpheny)-4,5,6,7-tetrahydro-3H-cyclopenta[b]pyridin-3-one (15b). Yield 65\%. m.p. $176-178^{\circ} \mathrm{C}$. IR (KBr) $v \mathrm{~cm}^{-1}: 1613(\mathrm{C}=\mathrm{N}), 1685(\mathrm{CO}), 3272(\mathrm{NH}) .{ }^{1} \mathrm{H}-\mathrm{NMR}$ (DMSO-d6): $\delta 1.79$

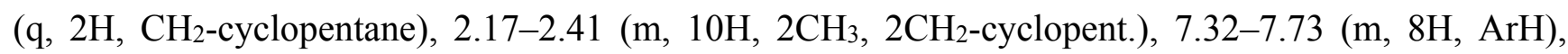
9.66 (bs, $2 \mathrm{H}, \mathrm{NH}_{2}, \mathrm{D}_{2} \mathrm{O}$ exchangeable). ${ }^{13} \mathrm{C}-\mathrm{NMR}$ (DMSO- $d_{6}$ ) $\delta$ ppm: 20.8, 22.4, 26.9, 47.1, 54.0 (saturated-C), 129.2, 130.4, 131.6, 133.8, 137.4, 140.9, $141.8(\operatorname{aromatic}+\mathrm{C}=\mathrm{N}), 192.0(\mathrm{C}=\mathrm{O})$. MS: $m / z 330\left[\mathrm{M}^{+}\right]$(37.6\%). Anal. Calc. for $\mathrm{C}_{22} \mathrm{H}_{22} \mathrm{~N}_{2} \mathrm{O}$ (330): C, 80.00; H, 6.67; N, 8.48; found: C, 79.97; H, 6.63; N, 8.48. 


\section{Conclusions}

In the present work, a series of some Schiff bases and novel fused heterocyclic derivatives 2-15 were synthesized using 2,3-diaryloxirane-2,3-dicarbonitriles 1a-c as starting materials. Some of newly synthesized compounds were screened against bacterial and fungal strains and most of the newly synthesized compounds showed high antimicrobial activities. The structures of the new compounds were elucidated using IR, ${ }^{1} \mathrm{H}-\mathrm{NMR},{ }^{13} \mathrm{C}-\mathrm{NMR}$ and mass spectroscopy.

\section{Acknowledgments}

The authors would like to extend their sincere appreciation to the Deanship of Scientific Research at King Saud University for its funding of this research through the Research Group project no. RGP-172.

\section{Author Contributions}

The listed authors contributed to this work as described in the following. Mohamed E. Azab gave the concepts of the work, interpreted the results and prepared the manuscript, Sameh A. Rizk, carried out the synthetic work, interpreted the results and prepared the manuscript and Abd El-Galil E. Amr interpreted the results and cooperated in the preparation of the manuscript. All authors read and approved the final manuscript.

\section{Conflicts of Interest}

The authors declare no conflict of interest

\section{References}

1. Muller, A.J.; Nishiyama, K.; Griffin, G.W.; Ishikawa, K.; Gibson, D.M. Reductive condensation of methyl arylglyoxylates. Direct synthesis of 2,3-bis(carbomethoxy)stilbene oxides and related systems. J. Org. Chem. 1982, 47, 2342-2352.

2. Li, Z.; Xu, J.; Niu, P.; Liu, C.; Yang, J.; Direct synthesis of 2,3-diaryloxirane-2,3-dicarbonitriles from aroyl chlorides using potassium hexacyanoferrate(II) as an eco-friendly cyanide source. Tetrahedron 2012, 68, 8880-8883.

3. Daidone, G.; Maggio, B.; Plescia, S.; Raffa, D.; Musiu, C.; Milia, C.; Perra, G.; Marongiu, M.E. Antimicrobial and antineoplastic activities of new 4-diazopyrazole derivatives. Eur. J. Med. Chem. 1998, 33, 375-382.

4. Singh, N.; Sangwan, N.K.; Dhindsa, K.S. Synthesis and fungitoxic activity of 5-aryl-1-formyl4,5-dihydro-3-(2-hydroxyphenyl)-1H-pyrazoles and their complexes. Pest Manag. Sci. 2000, 56, 284-288.

5. Daidone, G.; Raffa, D.; Plescia, F.; Maggio, B.; Roccaro, A. Synthesis of pyrazole-4carbohydrazide derivatives of pharmaceutical interest. Arkivoc 2002, xi, 227-235.

6. Migliara, O.; Plescia, S.; Diana, P.; di Stefano, V.; Camarda, L.; Dall'Olio, R. Synthesis and pharmacological evaluation of 7-substituted 1-ethyl-3,4,10-trimethyl-1,10-dihydro-11Hpyrazolo[3,4-c][1,6]benzodiazocin-11-one. A new ring system. Arkivoc 2004, v, 44-53. 
7. Bouabdallah, I.; M'Barek, L.A.; Zyad, A.; Ramdani, A.; Zidane, I.; Melhaoui, A. Anticancer effect of three pyrazole derivatives. Nat. Prod. Res. 2006, 20, 1024-1030.

8. Sato, N.; Jitsuoka, M.; Ishikawa, S.; Nagai, K.; Tsuge, H.; Ando, M.; Okamoto, O.; Iwaasa, H.; Gomori, A.; Ishihara, A.; et al. Discovery of substituted 2,4,4-triarylimidazoline derivatives as potent and selective neuropeptide Y Y5 receptor antagonists. Bioorg. Med. Chem. Lett. 2009, 19, 1670-1674.

9. Plachta, D.A.; Baranowski, A.M.; Laudy, A.E.; Starosciak, B.J.; Kleps, J. Synthesis of 1-\{4-[4(adamant-1-yl)phenoxymethyl]-2-(4-bromophenyl)-1,3-dioxolan-2-ylmethyl $\}$ imidazole with expected antifungal and antibacterial activity. Acta Pol. Pharm. Drug Res. 2007, 64, 535-540.

10. Owawiak, J.; Olender, D.; Zwolska, Z.; Augustynowicz-Kopec, E.; Zaprutko, L. Synthesis of 2,3dihydro-7-nitroimidazo[5,1-b]oxazoles as potential tuberculostatic agents. Acta Pol. Pharm. Drug Res. 2008, 65, 229-233.

11. Sharma, P.C.; Sharma, S.V.; Jain, S.; Singhd, D.; Suresh, B. Synthesis of some new isoxazoline derivatives as possible anti-candida agents. Acta Pol. Pharm. Drug Res. 2009, 66, 101-104.

12. Maczynski, M.; Zimecki, M.; Taraszkiewicz, M.; Ryng, S. Synthesis, immunological activity and computational study of 5-amino-3-methyl-4-isoxazolecarboxylic acid semicarbazides and thiosemicarbazides. Acta Pol. Pharm. Drug Res. 2008, 65, 543-549.

13. Maczynski, M.; Zimecki, M.; Ryng, S. A new class of isoxazole derivatives: The m 1-9 series of compounds with immunotropic activity. Acta Pol. Pharm. Drug Res. 2008, 65, 241-244.

14. Jain, M.; Nehra, S.; Trivedi, P.C.; Singh, R.V. Nematicidal, fungicidal and bactericidal activities of manganese(II) complexes with heterocyclic sulphonamide imines. Metal Based Drugs 2002, 9 , 53-60.

15. Patil, R.M. Synthetic, structural and biological properties of binuclear complexes with some schiff bases. Acta Pol. Pharm. Drug Res. 2007, 64, 345-353.

16. Rizk, S.A.; El-Hashash, M.A.; Mostafa, K.K. Utility of $\beta$-aroyl acrylic acids in heterocyclic synthesis. Egypt. J. Chem. 2008, 51, 611-621.

17. Rizk, S.A.; EL-Hashash, M.A.; Aburzeza, M.M. Utility of $p$-acetamidobenzoyl prop-2-enoic acid in the synthesis of new $\alpha$-amino acids and using them as building blocks in heterocyclic synthesis. Egypt. J. Chem. 2011, 54, 299-305.

18. Rizk, S.A. Utility of $E$-1-(4-acetamidobenzoyl)-2-oxirane carboxylic acid in synthesis some fused heterocycles and spiro compounds. Amer. J. Chem. 2011, 1, 65-72.

19. Azab, M.E.; Kassab, E.A.; El-Hashash, M.A.; Ali, R.S. Synthesis and antibacterial activity of some new 4(3H)quinazolin-4-one derivatives. Phos. Sulf. Silicon 2009, 184, 610-625.

20. Azab, M.E.; Youssef, M.M.; El-Bordany, E.A. Synthesis and antibacterial evaluation of novel heterocyclic compounds containing a sulfonamido moiety. Molecules 2013, 18, 832-844.

21. Khamees, H.; Jaleel, G.A.A.; Azab, M.E.; Mohamed, G.A.M.; Abdel-Aziz, T.A.; Eyada, H.A. Synthesis, characterization, anticancer, analgesic, and antiinflammatory activities of hitherto unknown thiazolo[3,2-a]pyridine and thiazolo[3,2-a]-1,8-naphthyridine derivatives. J. Atoms Mol. 2013, 3, 478-491.

22. Azab, M.E.; Amr, A.E. Synthesis of chiral linear and macrocyclic candidates: III. Synthesis and antimicrobial activity of linear tetrapeptide and macrocyclic pentapeptide Schiff bases. Russ. J. Gen. Chem. 2015, 85, 1513-1521. 
23. Said, A.S.; Amr, A.E.; El-Sayed, H.A.; Al-Omar, M.A.; Abdalla, M.M. Synthesized of some heterocyclic systems and their nucleoside of potent anti-inflammatory activities. Int. J. Pharm. 2015, 11, 502-507.

24. Fayed, A.A.; Al-Harb, N.; Amr, A.E.; Kalmoush, A.A.; Shadid, K.H.; Flefel, E.M. Synthesis, reactions, and pharmacological evaluations of some novel pyridazolopyridiazine candidates. $J$. Het. Chem. 2014, 51, 1770-1777.

25. Khalifa, N.M.; Al-Omar, M.A.; Amr, A.E.; Baiuomy, A.R.; Abdel Rahman, R.F. Synthesis and biological evaluation of some novel fused thiazolo[3,2-a]pyrimidines as potential analgesic and antiinflammatory agents. Russ. J. Bioorg. Chem. 2015, 41, 192-201.

26. Ouf, N.H.; Amr, A.E.; Sakran, M.I. Anticancer activity of some newly synthesized pyrano[2,3d] $[1,2,3]$ triazine derivatives using 1-(7-hydroxy-2,2-dimethyl-chroman-6-yl)ethanone as synthon. Med. Chem. Res. 2015, 24, 1514-1526.

27. Carson, C.F.; Riley, T.V. Antimicrobial activity of the major components of the essential oil of Melaleuca alternifolia. J. Appl. Bact. 1995, 78, 264-269.

28. Alam, M.S.; Lee, D.U.; Bari, L. Antibacterial and cytotoxic activities of Schiff base analogues of 4-aminoantipyrine. J. Korean Soc. Appl. Biol. Chem. 2014, 57, 613-619.

29. Cheng, Q.; Xu, X.; Wang, Q.; Zhang, L.; Lin, Q.; Zhang, J.; Yang, X. Synthesis and antibacterial activities of novel pyrazole Schiff bases and metal complexes. Chinese J. Org. Chem. 2009, 29, $1387-1391$.

Sample Availability: Samples of the compounds are available from the authors.

(C) 2015 by the authors; licensee MDPI, Basel, Switzerland. This article is an open access article distributed under the terms and conditions of the Creative Commons Attribution license (http://creativecommons.org/licenses/by/4.0/). 